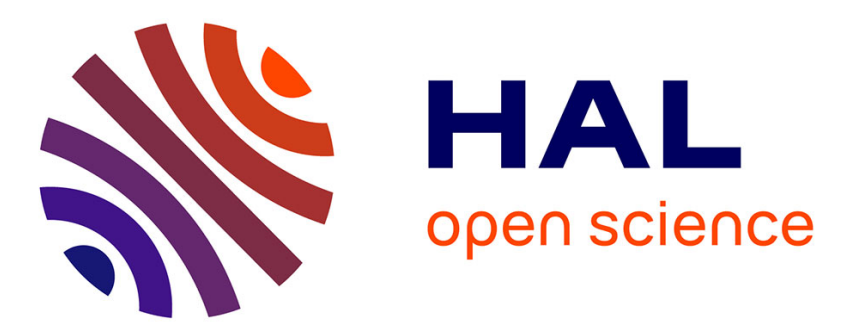

\title{
Langmuir Wave Decay in Inhomogeneous Solar Wind Plasmas: Simulation Results
}

\author{
C. Krafft, A. S. Volokitin, Vladimir Krasnoselskikh
}

\section{To cite this version:}

C. Krafft, A. S. Volokitin, Vladimir Krasnoselskikh. Langmuir Wave Decay in Inhomogeneous Solar Wind Plasmas: Simulation Results. The Astrophysical Journal, 2015, 809 (2), 176, 18 pp. 10.1088/0004-637X/809/2/176 . insu-01351972

\section{HAL Id: insu-01351972 \\ https://hal-insu.archives-ouvertes.fr/insu-01351972}

Submitted on 20 Nov 2019

HAL is a multi-disciplinary open access archive for the deposit and dissemination of scientific research documents, whether they are published or not. The documents may come from teaching and research institutions in France or abroad, or from public or private research centers.
L'archive ouverte pluridisciplinaire HAL, est destinée au dépôt et à la diffusion de documents scientifiques de niveau recherche, publiés ou non, émanant des établissements d'enseignement et de recherche français ou étrangers, des laboratoires publics ou privés. 


\title{
LANGMUIR WAVE DECAY IN INHOMOGENEOUS SOLAR WIND PLASMAS: SIMULATION RESULTS
}

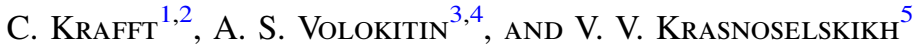 \\ ${ }^{1}$ Laboratoire de Physique des Plasmas, Ecole Polytechnique, F-91128 Palaiseau Cedex, France; catherine.krafft@u-psud.fr \\ ${ }^{2}$ Université Paris Sud, F-91405 Orsay Cedex, France \\ ${ }^{3}$ IZMIRAN, Troitsk, 142190, Moscow, Russia \\ ${ }^{4}$ Space Research Institute, 84/32 Profsoyuznaya Str., 117997 Moscow, Russia \\ ${ }^{5}$ Laboratoire de Physique et Chimie de l'Environnement et de l'Espace, 3A Av. de la Recherche Scientifique, F-45071 Orléans Cedex 2, France \\ Received 2015 February 9; accepted 2015 June 3; published 2015 August 21
}

\begin{abstract}
Langmuir turbulence excited by electron flows in solar wind plasmas is studied on the basis of numerical simulations. In particular, nonlinear wave decay processes involving ion-sound (IS) waves are considered in order to understand their dependence on external long-wavelength plasma density fluctuations. In the presence of inhomogeneities, it is shown that the decay processes are localized in space and, due to the differences between the group velocities of Langmuir and IS waves, their duration is limited so that a full nonlinear saturation cannot be achieved. The reflection and the scattering of Langmuir wave packets on the ambient and randomly varying density fluctuations lead to crucial effects impacting the development of the IS wave spectrum. Notably, beatings between forward propagating Langmuir waves and reflected ones result in the parametric generation of waves of noticeable amplitudes and in the amplification of IS waves. These processes, repeated at different space locations, form a series of cascades of wave energy transfer, similar to those studied in the frame of weak turbulence theory. The dynamics of such a cascading mechanism and its influence on the acceleration of the most energetic part of the electron beam are studied. Finally, the role of the decay processes in the shaping of the profiles of the Langmuir wave packets is discussed, and the waveforms calculated are compared with those observed recently on board the spacecraft Solar TErrestrial RElations Observatory and WIND.
\end{abstract}

Key words: instabilities - plasmas - solar wind - Sun: flares - turbulence - waves

\section{INTRODUCTION}

Direct observations of Langmuir waves in the solar wind and, in particular, in the source regions of type III solar bursts (e.g., Gurnett et al. 1981, 1992; Kellogg 1986; Kellogg et al. 1992, 2009; Ergun et al. 1998; Nulsen et al. 2007; Malaspina et al. 2010; Hess et al. 2011; Graham \& Cairns 2013, and references therein) and in the Earth's foreshock (e.g., Bale et al. 1996, 2000; Souček et al. 2005) show poor agreement with the predictions of the theory of Langmuir turbulence excited by electron beams in homogeneous plasmas (e.g., Vedenov et al. 1961, 1967; Musher et al. 1995). Moreover, many physical aspects revealed by the observations could not be described by analytical studies considering plasmas with simple density gradients or large density fluctuations (e.g., Breizman \& Ruytov 1970; Ryutov 1970). Thus, it is worth solving this conflict by taking into account the important role of plasma density irregularities in such plasmas and performing adequate numerical simulations, using kinetic or fluid approaches (e.g., Kontar \& Pecseli 2002; Krafft et al. 2013). On this basis it was shown on one hand that monotonic density gradients or randomly fluctuating density inhomogeneities are able to strongly modify the development and even the nature of the wave-particle and the wave-wave interactions at work and that on the other hand they can prevent the appearance of such nonlinear processes as wave decay, induced scattering, or modulation instability (but generally not beam quasilinear diffusion), whose descriptions are mostly based on theories developed in the frame of homogeneous plasmas (e.g., Zakharov 1972; Galeev et al. 1977; Rubenchik \& Shapiro 1993). Note that the low-frequency oscillations of both the plasma density and the ambient magnetic field can in principle significantly influence on the development of the beam instability and the waves' dynamics. However, since the solar wind magnetic field is weak (the ratio between the electron cyclotron and plasma frequencies satisfies $\omega_{c} / \omega_{p} \lesssim 10^{-2}$ ) and its effect on the dispersion of the plasma waves is negligible, one can assume that the fluctuating density irregularities only can have a significant impact on the Langmuir waves' and beams' dynamics. Unfortunately, only few direct measurements in the solar wind of such density fluctuations are available up to now, which can be used to determine their spectral properties as well as their average level (e.g., Celnikier et al. 1983, 1987; Kellogg et al. 1999; see also Malaspina et al. 2010).

One of the first simultaneous observations in the solar wind of long-wavelength density fluctuations (possibly ion sound (IS) waves) and intense Langmuir waves excited by electron beams associated with type III bursts was presented by Lin et al. (1986); they were interpreted as being due to nonlinear processes involving wave-wave interactions and in particular to Langmuir wave decay. Evidence or suspicion for threewaves' interactions in the source regions of type III solar radio bursts or in front of planetary bow shocks were reported in numerous papers; for example, the observations of waveforms in front of the Jovian bow shock were interpreted as beatings of Langmuir waves due to their interactions with IS waves or density fluctuations (Gurnett et al. 1981; Cairns \& Robinson 1992). More recent evidence for three-waves' interactions involving Langmuir waves in the electron foreshock region as well as in the source regions of type III solar bursts was reported by several authors (e.g., Lin et al. 1981, 1986; Robinson \& Newman 1991; Gurnett et al. 1993; Hospodarsky et al. 1994; Hospodarsky \& Gurnett 1995; Bale et al. 1996; Thejappa et al. 2003; Souček et al. 2005; Henri et al. 2009; Graham \& Cairns 2013, and references therein). Meanwhile, 
observations of electron fluxes or beams in the solar wind near the Earth's orbit were presented in other works (e.g., Lin et al. 1981; Ergun et al. 1998).

Recent studies of the wave activity measured on board satellites such as POLAR, ULYSSES, WIND, and the Solar TErrestrial RElations Observatory (STEREO) have revealed many new features of the Langmuir turbulence in much greater detail. For example, Langmuir wave decays during type III radio bursts are presented for 14 events observed by STEREO (Bougeret et al. 2008), where all wave packets were registered within a duration $\tau \simeq 130 \mathrm{~ms}$; the authors (Henri et al. 2009) argue that the threshold in the solar wind (Lin et al. 1986) of the decay instability of a Langmuir wave $\mathcal{L}$ into another Langmuir wave $\mathcal{L}^{\prime}$ and an IS wave $\mathcal{S}^{\prime}$ (i.e., $\mathcal{L} \rightarrow \mathcal{L}^{\prime}+\mathcal{S}^{\prime}$, hereafter called Langmuir electrostatic decay) is significantly exceeded. Moreover, Graham \& Cairns (2013) show that around $40 \%$ of the Langmuir waveforms observed by STEREO during type III solar radio bursts can be consistent with the occurrence of such decays, and even with one or more of their cascades. It is interesting to note here that the Langmuir wave profiles and spectra obtained using the INTERBALL-2 satellite's data (Burinskaya et al. 2004) in the inner regions of the Earth's magnetosphere are very similar to those observed in the solar wind type III regions or the foreshock; the explanation proposed by the authors is based on the weak turbulence theory of beam-excited Langmuir waves' scattering on the external IS turbulence.

In the papers reporting observations of Langmuir waves' decays in the solar wind, few arguments in favor of such nonlinear processes are mainly used (e.g., Lin et al. 1981, 1986; Robinson et al. 1993; Cairns \& Robinson 1995; Hospodarsky \& Gurnett 1995; Kellogg et al. 1999; Henri et al. 2009; Graham \& Cairns 2013 and references therein): (1) the existence of a double peaked structure in the Langmuir wave spectrum; (2) the simultaneous observation of intense Langmuir waves and IS waves or plasma density oscillations. In some cases, the frequencies of the observed IS waves fit well with the theoretical predictions of electrostatic decay based on beam and plasma measurements; (3) the estimate of the waves' intensities showing that the threshold of the Langmuir parametric instability in homogeneous plasmas is exceeded; (4) the tendency of the Langmuir waveforms observed to clump and burst, which exhibit modulation frequencies consistent with beatings between mother and daughter Langmuir waves. Usually these topics are not invoked all together simultaneously; moreover, in some cases, no signature of lowfrequency waves could be detected. Note finally that in order to conclusively identify an electrostatic decay, one should also verify the resonance conditions by a spectral analysis, the time occurrence of the waves by a wavelet analysis, and the phase correlations between the waves by a bicoherence analysis (e.g., Dudok de Wit \& Krasnoselskikh 1995; Thejappa et al. 2013).

When the threshold of the parametric instability is exceeded, intense Langmuir waves can decay into daughter waves according to the channel $\mathcal{L} \rightarrow \mathcal{L}^{\prime}+\mathcal{S}^{\prime}$, producing backscattered Langmuir waves $\mathcal{L}^{\prime}$ and IS waves $\mathcal{S}^{\prime}$ propagating in the same direction as the parent waves (in a one-dimensional (1D) description). If the $\mathcal{L}^{\prime}$ waves carry enough energy, a possible secondary decay cascade can occur according to the channel $\mathcal{L}^{\prime} \rightarrow \mathcal{L}^{\prime \prime}+S^{\prime \prime}$, producing in turn Langmuir waves $\mathcal{L}^{\prime \prime}$ propagating in the same direction as the mother waves $\mathcal{L}$ and IS waves $\mathcal{S}^{\prime \prime}$ propagating in the inverse direction. In principle, more cascades can occur until the decays become prohibited due to kinematic effects. The process $\mathcal{L} \rightarrow \mathcal{L}^{\prime}+\mathcal{S}^{\prime}$ allows the transfer of part of the wave energy from larger $k$-space regions to smaller ones, and in particular from regions where the beam resonantly excites waves (beam-driven mother waves) to regions (i) where such resonant phenomena are not possible (nonresonant domains where $k<0$, for example) or (ii) where the waves can resonantly damp and consequently release energy to beam particles and possibly accelerate them (when $k<k_{b}$, where $k_{b}=\omega_{p} / v_{b}$ and $v_{b}$ is the beam initial velocity). When the instability threshold is exceeded, the dynamics of the decay process depends notably on the energy of the parent Langmuir waves and on the efficiency of transferring it to the daughter waves, effects which are both influenced by the average level of randomly varying density fluctuations (and also by their wavelengths). In turn, decay processes can significantly influence the features of the Langmuir waveforms eventually observed and contribute to the shape of their modulations and accentuate their tendency to clump. Moreover the excitation of large-amplitude backscattered Langmuir waves may also lead to the appearance of transverse electromagnetic waves $\mathcal{T}$ near $2 \omega_{p}$ through the coalescence of two Langmuir waves, i.e., according to the channel $\mathcal{L}+\mathcal{L}^{\prime} \rightarrow \mathcal{T}\left(\omega_{p}\right.$ is the electron plasma frequency). This process is believed to be a first step for the generation of type III radio emissions at $2 \omega_{p}$; those can also be produced when a Langmuir wave decays in a transverse electromagnetic wave at $\omega_{p}$ and a low-frequency wave. Note that in the present study wave decay involving electromagnetic waves is not considered.

For all the reasons invoked above, it is essential to study Langmuir decay in solar wind plasmas using numerical simulations, especially because only a few analytical works exist dealing with inhomogeneous plasmas (e.g., Breizman \& Ruytov 1970; Ryutov 1970). In this view, several numerical simulations have been carried out in order to study the dynamics of wave decay in unmagnetized or weakly magnetized plasmas. Most of them were performed in the frame of the weak turbulence kinetic theory (e.g., Ziebell et al. 2001; Li et al. 2003; Henri et al. 2010), considering homogeneous plasmas and more rarely plasmas with inhomogeneities and in this latter case mainly in the form of monotonic gradients (e.g., Kontar \& Pecseli 2002). Particlein-cell (PIC) codes (e.g., Huang \& Huang 2008) or Vlasov codes (e.g., Umeda \& Ito 2008; Henri et al. 2010) have been used. Other simulations were performed in the frame of a fluid description using the Zakharov equations (e.g., Gibson et al. 1995). Among all these works, some were applied to study Langmuir wave decay in solar wind plasmas and in particular in the Earth's foreshock or in the source regions of type III solar bursts. Moreover, some were devoted to investigating not only wave decay but also other processes such as scattering off thermal ions, for example (see Cairns 2000, and references therein).

The present paper focuses on the problem of Langmuir wave decay in solar wind plasmas typical of type III bursts' source regions near $1 \mathrm{AU}$ where, as mentioned above, several observations report that such process can commonly occur; moreover, it is likely one of the most important mechanisms at work in these conditions. In such plasmas, randomly varying density fluctuations exist with average levels $\Delta n$ reaching up to several percents of the background plasma density (Celnikier 
et al. 1983, 1987; Kellogg et al. 1999); therefore, wave decay generally occurs simultaneously with other coupling effects between the fields and the density inhomogeneities, such as reflection, scattering, or refraction processes. For example, reflection phenomena on plasma irregularities can lead to the appearance of reflected waves of noticeable amplitudes which can couple to the beam-driven waves. In this case, electrostatic decay is less probable in plasmas with large average levels of density fluctuations $(\Delta n \gtrsim 0.03)$ than resonant wave-wave coupling, for which the resonance conditions are the same and the beatings between Langmuir waves also produce IS waves. In quasi-homogeneous plasmas, i.e., plasmas with density fluctuations of the order of or lower than $\Delta n \simeq 0.001-0.005$, where reflection effects are weak, "pure" electrostatic decay with daughter waves actually rising from the thermal noise can occur. Moreover, wave reflection on the gradients of the density fluctuations lead, near the reflection points of the density humps and wells, to wave energy focusing and to the appearance of short wave turbulence.

Thus, the aim of the paper is to understand what is the influence of plasma density inhomogeneities on the wave decay processes, that is, if they can favor or prevent their occurrence, and under what conditions. Note that at least two points will complicate the analysis of the simulation results and their comparison with theoretical works: (i) analytical studies were performed mainly for monochromatic waves (e.g., Sagdeev \& Galeev 1969; Zakharov et al. 1985), whereas our simulations involve broad wave packets; (ii) homogeneous or quasi-homogeneous plasmas were mostly considered even if some works include inhomogeneous plasmas with simple gradients or random oscillations (Breizman \& Ruytov 1970; Ryutov 1970; Escande \& de Genouillac 1978), whereas we take into account self-consistently randomly varying density inhomogeneities. Let us mention finally that in the frame of our theoretical model (see below) and according to the typical ratios of electron to ion temperatures in the solar wind, electrostatic decay overcomes the process of scattering off thermal ions (see also, e.g., Cairns 2000). Moreover, phenomena as modulational instability or collapse should not occur for our parameters due to their characteristic ranges of wavenumbers and high-frequency wave energy densities (Zakharov et al. 1985).

The simulation results are provided by a numerical code based on a Hamiltonian model (Krafft et al. 2013; Volokitin et al. 2013) where the self-consistent resonant interactions between beam electrons and Langmuir waves are described in a plasma involving density fluctuations for conditions relevant to solar type III observations at 1 AU (e.g., Ergun et al. 1998). Using the Zakharov's equations with an additional term representing the electron beam, the model takes into account strong preexisting and randomly varying density inhomogeneities and includes the low-frequency plasma response. The beam is modeled using a PIC description. Ponderomotive force effects are included, even if the density fluctuations do not result from strong turbulence phenomena. Contrary to the usual PIC approach where great numbers of particles are used to minimize the numerical noise, our model consists of dividing the electron distribution into two populations: (i) the bulk particles, forming the ambient plasma, which support the waves' propagation but do not interact resonantly with them; and (ii) the resonant beam electrons which exchange significant amounts of momentum and energy with the waves, and whose motion is solved by the Newton equations (e.g., O'Neil et al. 1971; Volokitin \& Krafft 2004; Zaslavsky et al. 2006). One of the advantages of this approach is to greatly reduce the number of particles used in the simulations and to solve their dynamics over long periods of time (e.g., Volokitin \& Krafft 2012).

\section{SUMMARY OF THE THEORETICAL MODEL}

Let us briefly summarize the theoretical model used and described in more detail in previous papers (Krafft et al. 2013; Volokitin et al. 2013). The interaction of Langmuir waves' packets with an electron beam in an inhomogeneous solar wind plasma can be studied in 1D geometry as most of the observed Langmuir wavefields are polarized along magnetic field lines (Ergun et al. 2008). The background plasma of density $n_{0}$ is described through the dielectric constant using linear theory, whereas only the resonant beam particles, of density $n_{b} \ll n_{0}$, interact with the electric Langmuir fields through the Landau resonance (e.g., O’Neil et al. 1971; Volokitin \& Krafft 2004; Zaslavsky et al. 2006).

The full Hamiltonian of the waves-particles-plasma system can be written as $\mathcal{H}=\mathcal{H}_{Z}+\mathcal{H}_{p}$, where $\mathcal{H}_{Z}$ is the Hamiltonian corresponding to the Zakharov equations (Zakharov 1972) without the beam source term

$$
\begin{aligned}
\mathcal{H}_{\mathrm{Z}}= & \int_{L} d z\left(\frac{\delta n}{2 n_{0}} \frac{|E|^{2}}{8 \pi}+\frac{3 \lambda_{\mathrm{D}}^{2}}{16 \pi}\left|\frac{\partial E}{\partial z}\right|^{2}\right. \\
& \left.+\frac{n_{0} m_{i}}{2}\left[c_{s}^{2}\left(\frac{\delta n}{n_{0}}\right)^{2}+\left(\frac{\partial \Phi}{\partial z}\right)^{2}\right]\right)
\end{aligned}
$$

and $\mathcal{H}_{p}$ takes into account the kinetic energy of the resonant particles and their energy of interaction with the waves

$$
\mathcal{H}_{p}=\sum_{p}\left(\frac{P_{p}^{2}}{2 m_{e}}-e \operatorname{Re} \sum_{k} i \frac{E_{k}}{k} e^{-i \omega_{p} t+i k z_{p}}\right) .
$$

The electric field is $\mathcal{E}=\operatorname{Re}\left(E(z, t) e^{-i \omega_{p} t}\right)$, where $E(z, t)=\sum_{k} E_{k}(t) e^{i k z}$ is the slowly varying field envelope, $|\partial E / \partial t| \ll \omega_{p}|E| ; \quad E_{k}$ is the Fourier component of $E ; z$ is the space coordinate and $k$ is the wave vector; $\omega_{k}=$ $\omega_{p}\left(1+3 k^{2} \lambda_{\mathrm{D}}^{2} / 2\right) \simeq \omega_{p}$ is the Langmuir wave frequency; $\omega_{p}$ and $\lambda_{\mathrm{D}}$ are the electron plasma frequency and Debye length; $\delta n$ is the ion density perturbation; $m_{e}$ and $m_{i}$ are the electron and ion masses, $u$ is the velocity of the ion population and $\Phi$ is the hydrodynamic flux potential, $u=\partial \Phi / \partial z ; T_{e}$ and $T_{i}$ are the electron and ion temperatures, with $T_{e} / T_{i} \gtrsim 3 ; c_{s}$ is the ion acoustic velocity; $v_{p}, z_{p}$ and $P_{p}=m_{e} v_{p}$ are the velocity, the position, and the generalized momentum of the electron $p$, respectively; $L$ is the size of the system and $N=L n_{b}$ is the number of beam particles; $-e<0$ is the electron charge.

The following Hamilton equations

$$
\begin{gathered}
\frac{1}{8 \pi \omega_{p}} \frac{\partial E}{\partial t}=-i \frac{\delta \mathcal{H}}{\delta E^{*}}, \quad n_{0} m_{i} \frac{\partial \Phi}{\partial t}=-\frac{\delta \mathcal{H}}{\delta\left(\frac{\delta n}{n_{0}}\right)}, \\
v_{p}=\frac{d z_{p}}{d t}=\frac{\partial \mathcal{H}}{\partial P_{p}}, \quad \frac{d P_{p}}{d t}=-\frac{\partial \mathcal{H}}{\partial z_{p}}
\end{gathered}
$$


where $\delta$ is the functional derivative, provide the complete set of the model's equations, that is

$$
\begin{gathered}
m_{e} \frac{d v_{p}}{d t}=-e \mathcal{E}\left(z_{p}, t\right)=-e \operatorname{Re} \sum_{k} E_{k} e^{i k z_{p}-i \omega_{k} t} \\
i \frac{\partial E}{\partial t}+\frac{3 \lambda_{\mathrm{D}}^{2}}{2} \omega_{p} \frac{\partial^{2} E}{\partial z^{2}}-\omega_{p} \frac{\delta n}{2 n_{0}} E=4 \pi i e n_{b} \sum_{k} \frac{\omega_{p}}{k} J_{k} e^{i k z} \\
\left(\frac{\partial^{2}}{\partial t^{2}}-c_{s}^{2} \frac{\partial^{2}}{\partial z^{2}}\right) \frac{\delta n}{n_{0}}=\frac{\partial^{2}}{\partial z^{2}} \frac{|E|^{2}}{16 \pi m_{i} n_{0}} \\
\frac{\partial}{\partial t} \frac{\delta n}{n_{0}}=-\frac{\partial u}{\partial z}
\end{gathered}
$$

where

$$
J_{k}=\frac{1}{N} \sum_{p} e^{i \omega_{k} t-i k z_{p}}
$$

In the Fourier space, Equations (6)-(8) can be written as

$$
\begin{gathered}
i\left(\frac{\partial}{\partial t}-\gamma_{k}^{(e)}\right) E_{k}=\frac{3}{2} \omega_{p} k^{2} \lambda_{\mathrm{D}}^{2} E_{k}+\frac{\omega_{p}}{2}(\rho E)_{k}+i \frac{4 \pi e \omega_{p} n_{b}}{k} J_{k} \\
\frac{\partial u_{k}}{\partial t}-2 \gamma_{k}^{(i)} u_{k}=-i k c_{s}\left(\rho_{k}+\frac{\left(|E|^{2}\right)_{k}}{16 \pi m_{i} n_{0} c_{s}^{2}}\right) \\
\frac{\partial}{\partial t} \rho_{k}=-i k c_{s} u_{k}
\end{gathered}
$$

where we introduced $\rho=\delta n / n_{0}$ and added the damping factors $\gamma_{k}^{(e)}=-\operatorname{Im} \varepsilon_{k}^{(e)} /\left(\partial \operatorname{Re} \varepsilon_{k}^{(e)} / \partial \omega_{k}\right)$ and $\gamma_{k}^{(i)}=-\operatorname{Im} \varepsilon_{k}^{(i)} /$ $\left(\partial \operatorname{Re} \varepsilon_{k}^{(i)} / \partial \omega_{k}\right) \quad\left(\varepsilon_{k}^{(e)}\right.$ and $\varepsilon_{k}^{(i)}$ are the electronic and ionic dielectric constants) in order to take into account damping effects on the electrons and the ions, respectively; $u_{k}$ and $\rho_{k}$ are the Fourier components of $u$ and $\rho$, respectively. Indeed, even if in the present description the background plasma is not modeled using individual particles, nonthermal tails of its velocity distribution can play a role as they lie in the velocity range where wave-particle interaction processes take place.

\section{LANGMUIR WAVE DECAY: SIMULATION RESULTS}

Let us present simulation results on wave-wave interaction processes occurring in the course of Langmuir turbulence generated by weak electron beams associated with type III bursts in solar wind plasmas with random density fluctuations $\delta n$. Those are supposed to have typical scales $\lambda_{n}$ much larger than the plasmons' wavelengths, of the order or more than several hundreds of Debye lengths $\lambda_{\mathrm{D}}$, and average amplitudes $\Delta n=\sqrt{\left\langle\left(\delta n / n_{0}\right)^{2}\right\rangle}$ ranging from roughly 0.001 to $0.05\left(n_{0}\right.$ is the background plasma density). The study is performed using a 1D numerical code based on a theoretical model which incorporates the dynamics of an electron beam inside the Zakharov equations (Krafft et al. 2013; Volokitin et al. 2013; Krafft \& Volokitin 2014). The randomly varying density fluctuations are preexisting at the initial state (they are not created by the ponderomotive term in the low-frequency equation) and evolve self-consistently in time and space with the high-frequency waves and the electron beam.
Calculations are carried out for parameters typical for solar type III beams and plasmas near 1 AU (e.g., Ergun et al. 1998); the beam drift and thermal velocities $v_{b}$ and $\Delta v_{b}$ range from $10 \lesssim v_{b} / v_{T} \lesssim 50$ and $0.05 \lesssim \Delta v_{b} / v_{b} \lesssim 0.1 ; v_{T}$ is the electron thermal velocity of the background plasma. The beam density $n_{b}$ is much smaller than the plasma density $n_{0} \simeq 510^{6} \mathrm{~m}^{-3}$, i.e., $510^{-6} \lesssim n_{b} / n_{0} \lesssim 510^{-5}$. The ambient plasma temperature and the electron Debye length are around $T_{e} \simeq 10-20 \mathrm{eV}$ and $\lambda_{\mathrm{D}} \sim 15 \mathrm{~m}$. Below $\omega_{p} t, z / \lambda_{\mathrm{D}}, \delta n / n_{0}$ $|E|^{2} / 4 \pi n_{0} T_{e}$ and $v / v_{T}$ are the normalized values of time, space, density, Langmuir energy density, and velocity.

Waves and particles are initially distributed in a periodic simulation box of size $L=10000-30000 \lambda_{\mathrm{D}}$. The beam velocity distribution is modeled by a Maxwellian function and the resonant electrons are distributed uniformly in space. Even if the resonant electrons travel several times along the periodic simulation box during simulations performed over long time periods, one can consider that at each of their passages they interact with Langmuir waves with new spectra and phases, as the timescale for a significant variation of the Langmuir turbulence is shorter than the electrons' time of travel through the box. Initially, 1024-2048 plasma waves of random phases and small amplitudes are distributed in Fourier space, with wavevectors $-k_{\max }<k<k_{\max }$, where $k_{\max } \lambda_{\mathrm{D}} \simeq 0.2-0.3$. The space and time resolutions are, depending on the simulations, around $2-10 \lambda_{\mathrm{D}}$ and $0.01-0.02 \omega_{p}^{-1}$, respectively.

We first study the process of electrostatic Langmuir wave decay $\mathcal{L} \rightarrow \mathcal{L}^{\prime}+\mathcal{S}^{\prime}$ in a plasma with a small average level of density fluctuations, i.e., $\Delta n \simeq 0.001$; the initial density spectrum is typically a Gaussian with a finite temperature. Then, we focus our attention on the development of such decay in inhomogeneous plasmas with larger $\Delta n$, in order to show that it can also occur in such conditions, but in a modified way.

\subsection{Langmuir Wave Decay in a Quasi-homogeneous Plasma}

Let us first consider simulations performed for a plasma with a small average level of density fluctuations, i.e., $\Delta n \simeq 0.001$. Figure 1 shows the growth with time of the normalized spectral energy density $W_{L}=\sum_{k}\left|E_{k}\right|^{2}$ of the Langmuir waves excited by the beam and the corresponding time evolution of the beam velocity distribution $f(v) ; W_{L}$ grows until saturation near $\omega_{p} t \simeq 60000$, whereas $f(v)$ widens toward lower velocities, asymptotically forming a plateau with a vanishing slope $\partial f(v) / \partial v \simeq 0$. Indeed, as $\Delta n$ is very below the threshold $\sim 3\left(v_{T} / v_{b}\right)^{2}$ determined in our previous works (Krafft et al. 2013; Volokitin et al. 2013), the dynamics of the system roughly presents the same features as those described by the quasilinear theory of the weak turbulence in homogeneous plasmas (Vedenov \& Ryutov 1975, p. 3; see also Volokitin et al. 2013) or other close models (e.g., O'Neil et al. 1971; Volokitin \& Krafft 2004; Krafft et al. 2005, 2010; Krafft \& Volokitin 2006, 2010; Zaslavsky et al. 2006, 2007; Krafft \& Volokitin 2013). In this case it was shown (e.g., Krafft et al. 2013) that the density inhomogeneities weakly influence the development of the beam instability and that the main features observed are (i) the formation of a plateau in the velocity distribution function $f(v)$ at asymptotic times, (ii) the dependence of the wave spectral energy density, scaling as $\left|E_{k}\right|^{2} \propto\left(n_{b} / n_{0}\right)\left(\omega_{k} / k\right)^{4}$ in the velocity domain above the thermal region where the beam can excite Langmuir waves, 

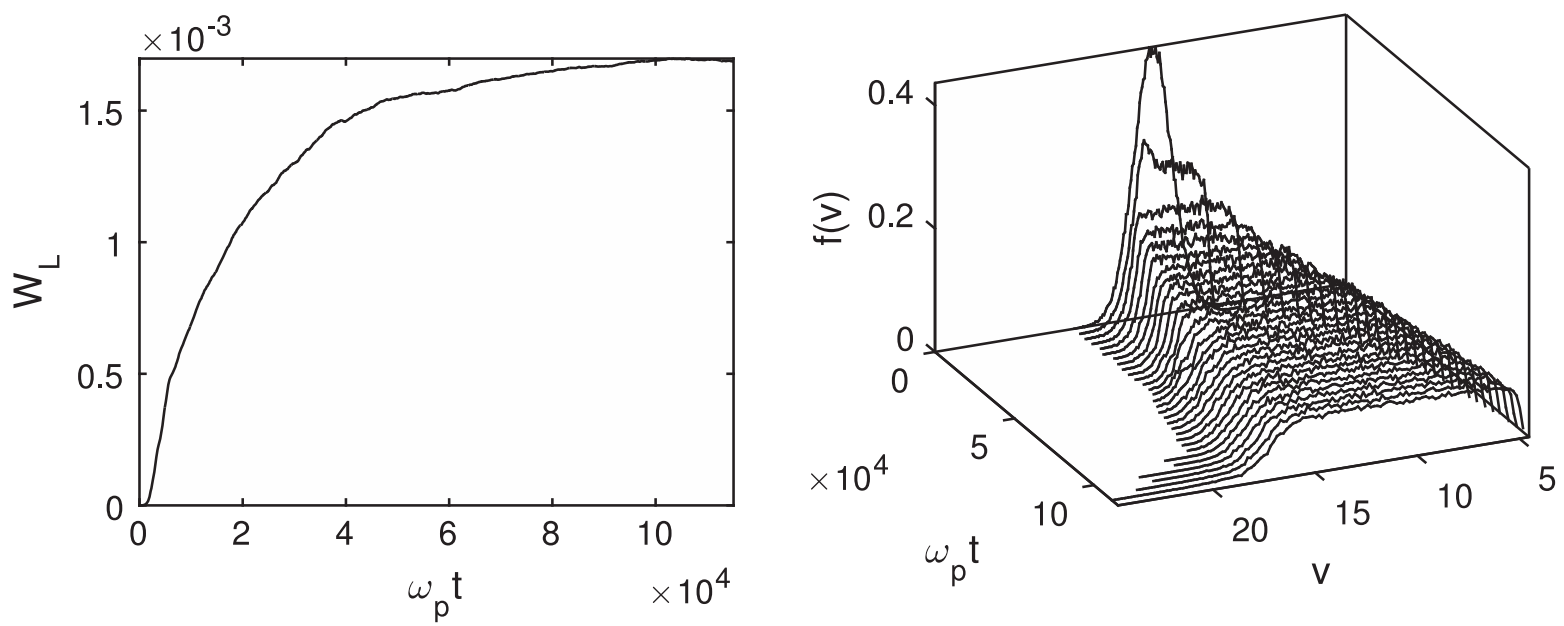

Figure 1. Left: time variation of the normalized spectral energy density $W_{L}$ of the Langmuir waves. Right: time variation of the beam velocity distribution $f(v)$; the velocity $v$ is normalized by $v_{T}$. The main parameters are the following: $n_{b} / n_{0}=510^{-5}, v_{b} / v_{T}=14, \Delta n \simeq 0.001, L=10000 \lambda_{\mathrm{D}}$.
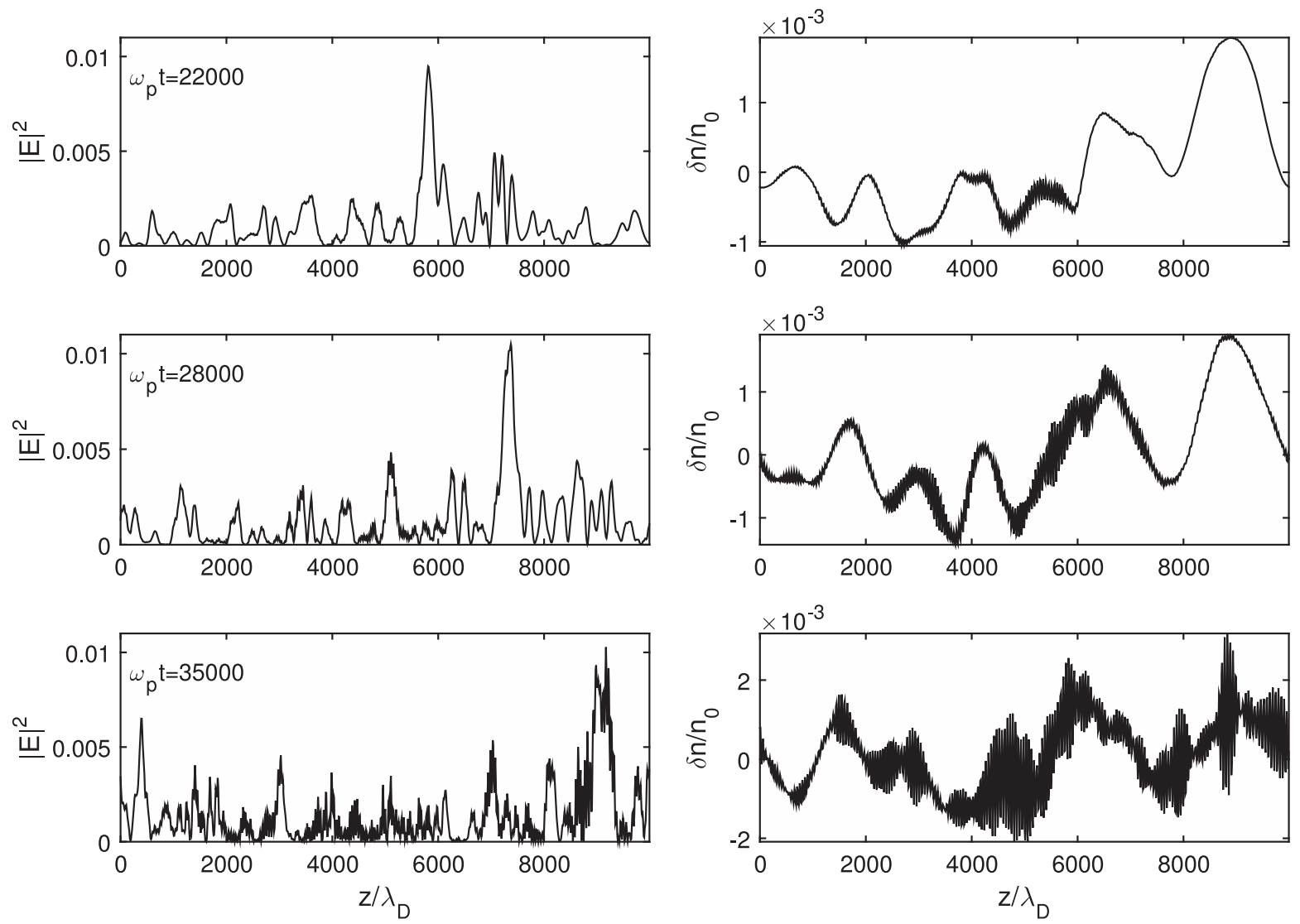

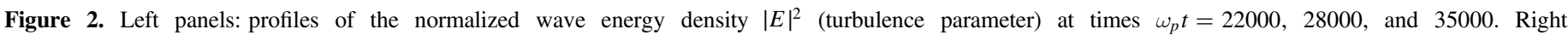

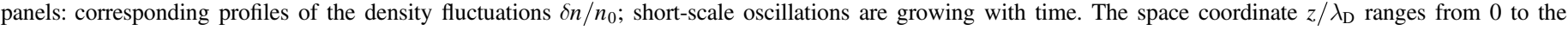
size $L$ of the simulation box. Parameters are the same as in Figure 1.

and (iii) a very small amount of accelerated beam electrons if $\Delta n$ is not vanishing (e.g., Krafft et al. 2013).

Up to the time $\omega_{p} t \simeq 30000$, most features of the system's evolution are in agreement with the predictions of the quasilinear theory of Langmuir waves. However, for $\omega_{p} t \gtrsim 30000$, short-wavelength density oscillations, which have been identified as IS waves, appear and grow with time along the full length $L$ of the system, as shown by Figure 2 which presents the Langmuir wave energy density $|E|^{2}$ and the density fluctuations $\delta n / n_{0}$ as a function of the space coordinate $z / \lambda_{\mathrm{D}}$ at three different times $\omega_{p} t=22000,28000$, and 35000. The short-wavelength density perturbations $\delta n_{i s} / n_{0}$ (see Equation (12)) present rather large amplitudes whereas the Langmuir wave packets reveal energy densities $|E|^{2}$ peaking up to around 0.01. To study these fluctuations in more detail and separate them from the background long-wavelength fluctuations $\delta n / n_{0}$, we filter $\delta n$ and the normalized plasma velocity $u$, which consists of removing all the Fourier harmonics $\delta n_{k}$ and 

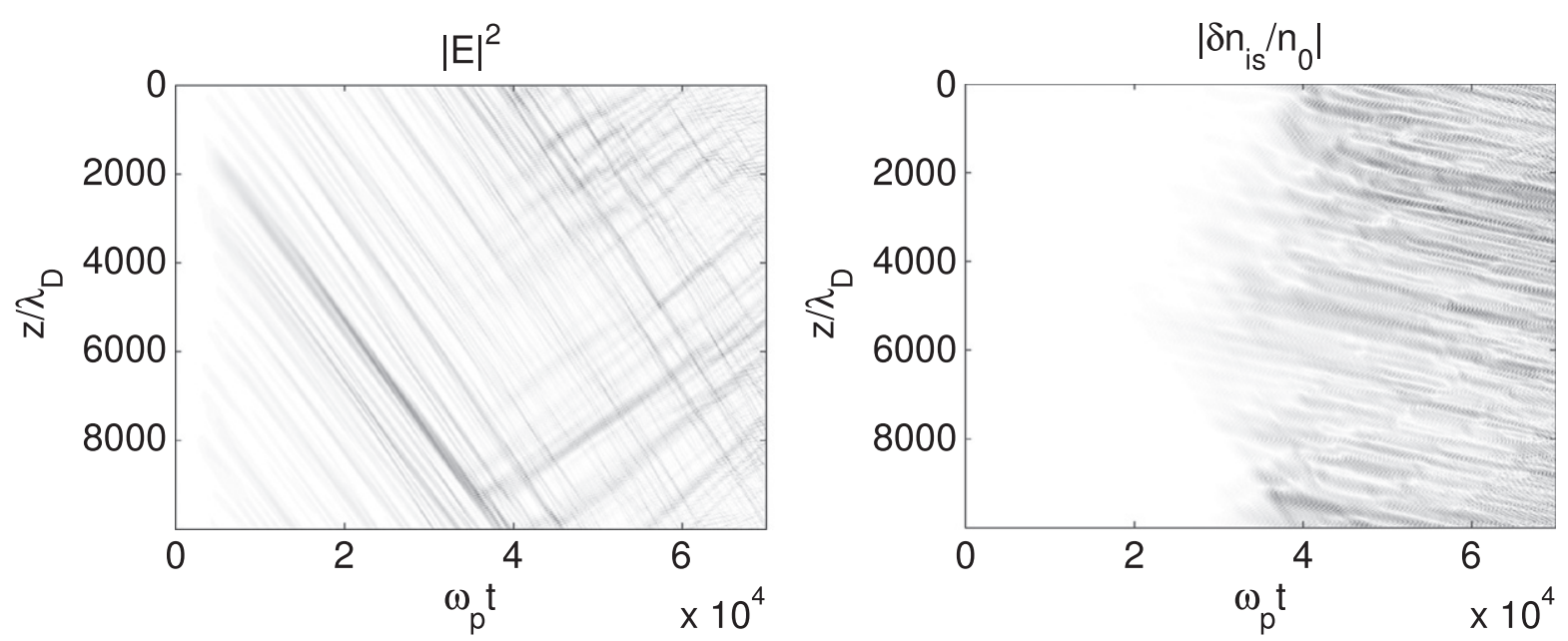

Figure 3. Left: space and time variations of the normalized Langmuir energy density $|E|^{2}$. Right: space and time variations of the corresponding short-scale density fluctuations $\left|\delta n_{i s} / n_{0}\right|$. Parameters are the same as in Figure 1.

$u_{k}$ with $k<k_{*} \sim 2-3 k_{b}\left(k_{b}=\omega_{p} / v_{b}\right)$; we obtain the shortwavelength density and plasma velocity in the form

$$
\delta n_{i s}=\sum_{k>k_{*}} \delta n_{k}(t) e^{i k z}, \quad u_{i s}=\sum_{k>k_{*}} u_{k}(t) e^{i k z} .
$$

Note that it is not essential to determine the exact value of $k_{*}$ and that we remove the parasitic oscillations that remain after this operation at the edges of the chosen space portion where the filtering is applied. Despite its arbitrariness, this procedure constitutes an effective method for analyzing the properties of the excited IS oscillations.

Using Equation (12), we present in Figure 3 the time and space variations of the turbulence parameter $|E|^{2}$ and the shortscale oscillations $\delta n_{i s} / n_{0}$ over the full time range of the simulation. The dark lines traveling downward (Figure 3, left) correspond to Langmuir waves propagating along the beam direction with the group velocity $v_{g} / v_{T} \simeq 3 k_{b} \lambda_{\mathrm{D}} \simeq 0.2$ $\left(k_{b} \lambda_{\mathrm{D}} \simeq 0.07\right.$ ). At $\omega_{p} t \gtrsim 30000$, IS waves appear (Figure 3, right) that propagate in the beam direction with a velocity around the normalized IS velocity $c_{S} / v_{T}$. Simultaneously (at $\left.\omega_{p} t \gtrsim 30000\right)$, Langmuir waves propagating in the direction opposite to the beam with the group velocity $-v_{g} / v_{T} \simeq-0.2$ can be observed. This picture is in full agreement with the development of a nonlinear decay process during which a Langmuir wave $\mathcal{L}\left(\omega_{L}, k_{L}\right)$ transfers energy to a counterpropagating Langmuir wave $\mathcal{L}^{\prime}$ of frequency $\omega_{L^{\prime}}$ and wavenumber $k_{L^{\prime}}<0$ and an IS wave $\mathcal{S}^{\prime}\left(\omega_{S^{\prime}}, k_{S^{\prime}}>0\right)$ ). During this interaction the conditions of parametric resonance $\omega_{L}=$ $\omega_{L^{\prime}}+\omega_{S^{\prime}}$ and $k_{L}=k_{L^{\prime}}+k_{S^{\prime}}$ should be verified. As is well known from the theory developed in homogeneous plasmas (Kadomtsev 1965; Nicholson \& Goldman 1978), those are satisfied if $k_{L^{\prime}} \simeq k_{0}-k_{L}$ and $k_{S^{\prime}} \simeq 2 k_{L}-k_{0}$, with $k_{L} \simeq k_{b}$ and $k_{0} \lambda_{\mathrm{D}}=2 c_{s} / 3 v_{T} \simeq 0.03$; corresponding wave dispersions are $\omega_{L} \simeq \omega_{p}\left(1+3 k_{L}^{2} \lambda_{\mathrm{D}}^{2} / 2\right)$ and $\omega_{S} \simeq c_{S} k_{S^{\prime}}$. We show below that in the present case these resonance conditions are fulfilled.

Let us study the time evolution of the Langmuir and IS waves' spectra. To distinguish between the IS waves propagating in the positive and the negative directions (i.e., in the direction of the beam propagation and opposite to it, respectively), we calculate the spectra of the Riemann invariants $R_{+}=\rho+u$ and $R_{-}=\rho-u$, where $\rho=\delta n / n_{0}$. In the absence of Langmuir waves, $R_{+}$is conserved along the line $d z / d t=c_{s}$ for IS waves propagating in the positive direction (increasing $z$ in Figure 3 ) and $R_{-}$is conserved along the line $d z / d t=-c_{s}$ for IS waves propagating in the negative direction (decreasing $z$ in Figure 3). Thus, the spectrum of the energy density of the IS waves can be represented by $S_{k}$, with $S_{k}=\left[(\rho+u)^{2}\right]_{k}$ for $k \geqslant 0$ and $S_{k}=\left[(\rho-u)^{2}\right]_{k}$ for $k<0: S_{k}$ is the spectrum of the IS waves with $k \geqslant 0$ propagating in the positive direction and of the IS waves with $k<0$ propagating in the negative direction.

The spectra of the Langmuir and the IS waves' energy densities $\left|E_{k}\right|^{2}$ and $S_{k}$ are calculated in Figure 4 for the same times as the profiles of $|E|^{2}$ and $\delta n / n_{0}$ in Figure 2. The main peak centered near $k \lambda_{\mathrm{D}} \simeq k_{b} \lambda_{\mathrm{D}} \simeq 0.07$ in the $\left|E_{k}\right|^{2}$ spectrum corresponds to waves $\mathcal{L}$ excited by the beam instability near the most unstable mode around $\omega_{k} / k \simeq v_{b}-\Delta v_{b} \simeq 12.7 v_{T}$ (Figure 4, upper left); note that $\left|E_{k}\right|^{2}$ broadens with time toward higher $k \gtrsim k_{b}$ (i.e., lower phase velocities $\omega_{k} / k<v_{b}$ ); no waves with small $k>0$ are visible as wave scattering on the density fluctuations $\delta n$ is very weak. A second peak (waves $\mathcal{L}^{\prime}$ ) appears at $\omega_{p} t \simeq 22000$ near $k \lambda_{\mathrm{D}} \simeq-0.04$; it grows with time, eventually reaching the same amplitude as the other one at $k \lambda_{\mathrm{D}} \simeq 0.07$. Meanwhile, IS waves $\mathcal{S}^{\prime}$ are excited around $k \lambda_{\mathrm{D}} \simeq 0.11$, as shown by the $S_{k}$ spectrum at $\omega_{p} t \simeq 22000$ (Figure 4, lower left); this peak grows in correspondence with that at $k \lambda_{\mathrm{D}} \simeq-0.04$ (note that the peak near $k=0$ in the $S_{k}$ spectrum corresponds to the initial density oscillations and should not be considered here). One can identify the first cascade of the decay process $\mathcal{L} \rightarrow \mathcal{L}^{\prime}+\mathcal{S}^{\prime}$. Indeed, the theory of three-waves' resonant decay in a homogeneous plasma predicts that for a parent Langmuir wave at $k_{L} \lambda_{\mathrm{D}} \simeq 0.07$ we should get $k_{L^{\prime}} \lambda_{\mathrm{D}} \simeq\left(k_{0}-k_{L}\right) \lambda_{\mathrm{D}} \simeq-0.04$ and $k_{S^{\prime}} \lambda_{\mathrm{D}} \simeq\left(2 k_{L}-k_{0}\right) \lambda_{\mathrm{D}} \simeq 0.11$, which fits very well with the observations (Figure 4). Further (at $\omega_{p} t \gtrsim 65000$, not shown here), a second cascade of decay occurs, i.e., $\mathcal{L}^{\prime} \rightarrow \mathcal{L}^{\prime \prime}+\mathcal{S}^{\prime \prime}$, providing Langmuir waves with $k_{L^{\prime \prime}} \lambda_{\mathrm{D}} \simeq$ $\left(k_{L}-2 k_{0}\right) \lambda_{\mathrm{D}} \simeq 0.02$ and IS waves near $k_{S^{\prime \prime}} \lambda_{\mathrm{D}}=$ $\left(-2 k_{L}+3 k_{0}\right) \lambda_{\mathrm{D}} \simeq-0.05$, that propagate in the direction opposite to the beam drift, i.e., with a group velocity $-c_{S} / v_{T}$. The secondary decay process is weaker than the first one and the amplitudes of the involved IS waves are smaller. Nevertheless, the cascading process occurs two times, as expected by the theory (no further cascades as $k_{L} / k_{0}<3$ ); unless damping 

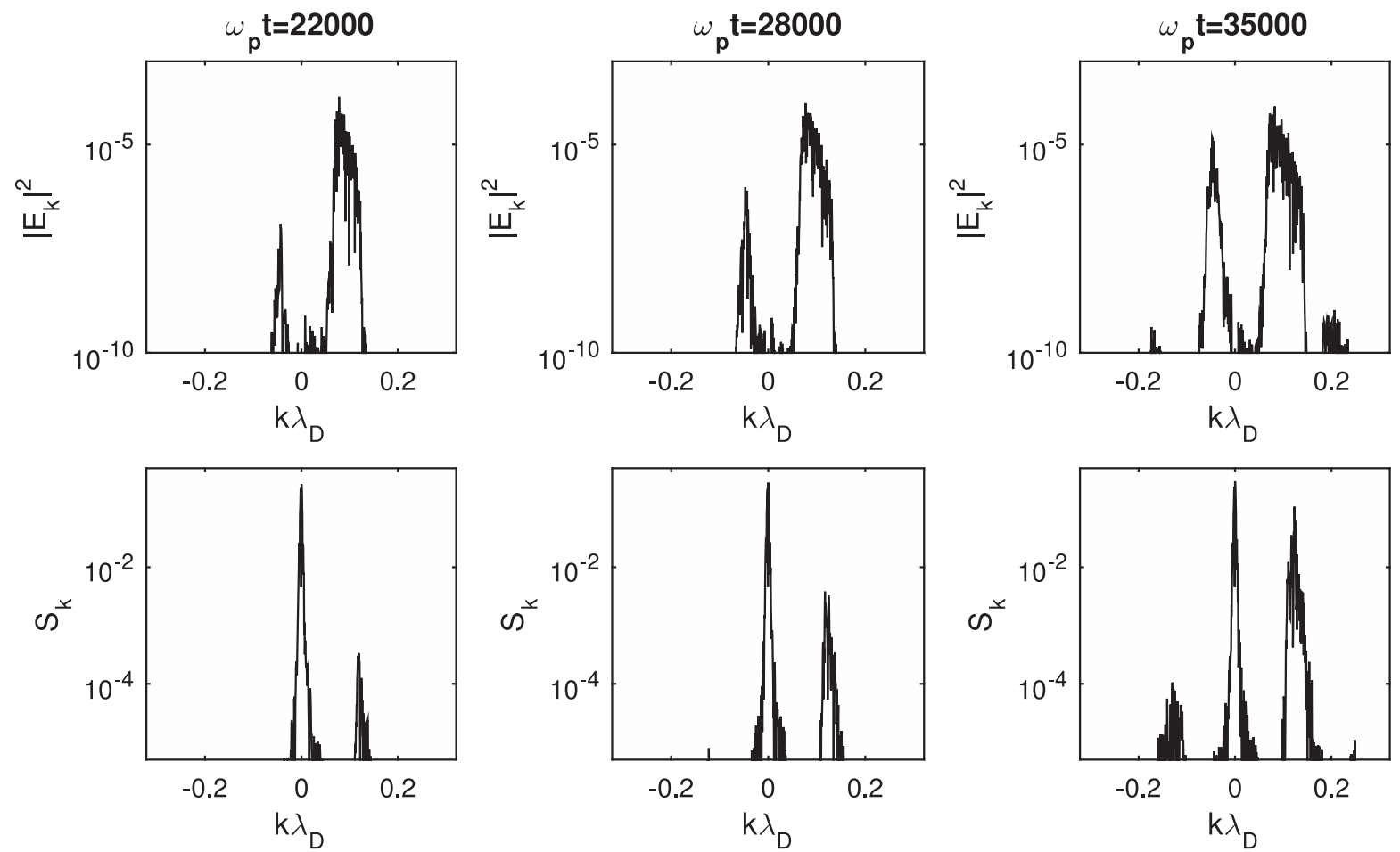

Figure 4. Upper panels: spectra $\left|E_{k}\right|^{2}$ of the Langmuir waves (in logarithmic scales) for the same instants of time as in Figure 2, i.e., $\omega_{p} t=22000,28000$, and 35000 , as a function of $k \lambda_{\mathrm{D}}$. Lower panels: Corresponding spectra (in logarithmic scales) of the energy density $S_{k}$ of the IS waves with $k \geqslant 0$ propagating in the positive direction and of the IS waves with $k<0$ propagating in the negative direction. Parameters are the same as in Figure 1.

of waves is included (what is not the case here), there is no threshold for such decay processes.

The energy of the IS waves starts to grow when the Langmuir turbulence is almost saturated; thus, one can expect to observe a clear linear stage of the decay instability. Indeed, as shown in Figure 5, the energy $\int_{L}\left|\delta n_{i s} / n_{0}\right|^{2} d z / L$ of the short-scale IS oscillations (integrated over the full simulation box) grows exponentially within the time range $21000 \lesssim \omega_{p} t \lesssim 37000$, whereas the corresponding Langmuir energy $\int_{L}|E|^{2} d z / L$ changes only slightly. The growth rate $\Gamma$ of the IS energy can be estimated as

$$
\frac{\Gamma}{\omega_{p}} \simeq \frac{1}{2} \Delta \ln \left(\int_{L}\left|\frac{\delta n_{i s}}{n_{0}}\right|^{2} \frac{d z}{L}\right) / \Delta\left(\omega_{p} t\right) \simeq 2-310^{-4},
$$

which is indeed smaller than but close to the maximum growth rate of the decay instability given by Galeev et al. (1975) for monochromatic waves in homogeneous plasmas

$$
\frac{\gamma_{\mathrm{D}}}{\omega_{p}} \simeq \frac{1}{2} \sqrt{\frac{\omega_{S}}{\omega_{p}} \frac{|E|^{2}}{16 \pi n_{0} T_{e}}} .
$$

Here $\omega_{S}$ is the frequency of the IS waves; then, as $\omega_{S} / \omega_{p} \simeq 2 c_{s} k_{b} / \omega_{p} \simeq 0.006$ and $|E|^{2} / 16 \pi n_{0} T_{e} \simeq 10^{-4}$ (average value) we get $\gamma_{D} \simeq 410^{-4} \omega_{p}$. The uncertainty in the determination of the average value of $|E|^{2} / 16 \pi n_{0} T_{e}$ in Equation (14) and the fact that $\gamma_{\mathrm{D}}$ is calculated for monochromatic waves in homogeneous plasmas can explain the difference between $\Gamma$ and the theoretical value $\gamma_{\mathrm{D}}$. Note also that, as expected, the nonlinear growth rates $\Gamma$ and $\gamma_{\mathrm{D}}$ are

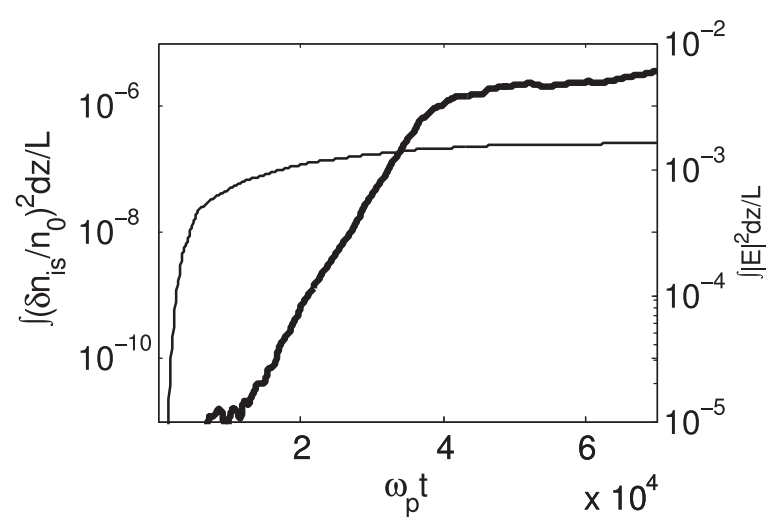

Figure 5. Time variation of the energy $\int_{L}\left|\delta n_{i s} / n_{0}\right|^{2} d z / L$ of the short-scale IS oscillations (thick line and left axis) and of the energy $\int_{L}|E|^{2} d z / L$ of the Langmuir waves (thin line and right axis) integrated over the full system's length $L$, in logarithmic scales. Parameters are the same as in Figure 1.

significantly smaller than the typical minimum frequency $\omega_{S}=0.006$ of the waves involved in the decay, ensuring that the collective response of the IS waves can take place as well as the exchanges of energy during the wave-wave coupling.

Finally, we can conclude that our simulations, which were carried out for a small average level $\Delta n \simeq 0.001$ of density fluctuations, are consistent with the theory of weak turbulence for Langmuir waves resonantly interacting with IS waves through the channel $\mathcal{L} \rightarrow \mathcal{L}^{\prime}+\mathcal{S}^{\prime}$. Note that, obviously, the beam instability does not excite a monochromatic wave but a broad wave spectrum. Then, as shown by the above figures, many waves of this wide packet can decay, producing a broad spectrum of daughter waves. The fastest Langmuir decay occurs for waves with the largest field amplitudes, as shown by 

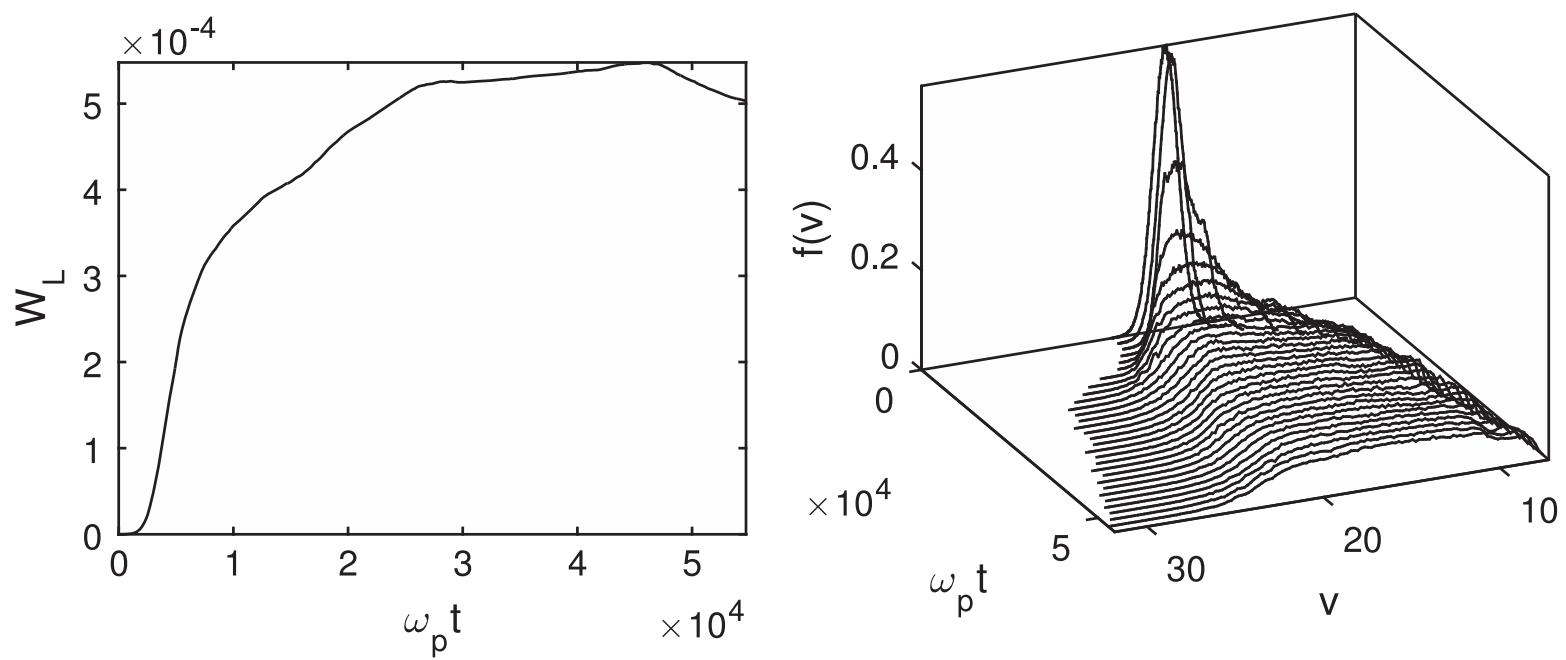

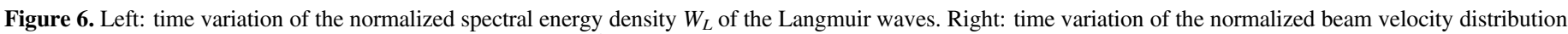
$f(v)$. The main parameters are the following: $n_{b} / n_{0}=210^{-5}, v_{b} / v_{T}=18, \Delta n \simeq 0.01, L=32000 \lambda_{\mathrm{D}}$.

Equation (14), and the waves with larger $k$ can undergo more decay cascades than those with smaller $k$. After the occurrence of several cascades, the Langmuir energy can be accumulated within the region $-k_{0} / 2<k<k_{0} / 2$, where waves cannot continue to decay but where the process of scattering off ions can become effective (see e.g., Cairns 2000; Kontar \& Pecseli 2002). However, this problem is not considered here.

The influence of the background density inhomogeneities on the system's dynamics becomes significant only when $\Delta n$ approaches or exceeds the threshold $3\left(v_{T} / v_{b}\right)^{2}$, as shown by our previous works (Krafft et al. 2013; Volokitin et al. 2013) and the study we present herein concerning wave decay. We will consider in details successively two cases, when $\Delta n=0.01$ and $\Delta n=0.02$.

\subsection{Langmuir Wave Decay in Inhomogeneous Plasmas with Random Density Fluctuations}

When the average level of density fluctuations $\Delta n$ becomes of the order or larger than the threshold $3\left(v_{T} / v_{b}\right)^{2}$, the dynamics of the system is strongly influenced by the scattering of the waves on the density inhomogeneities. Indeed, the wave-particle resonance conditions are violated due to the random variations of the waves' phase velocities $\omega_{k} / k \simeq \omega_{p}(z) / k ;$ as a consequence, and compared to the homogeneous plasma case, the rate of growth of the Langmuir wave energy emitted during the bump-on-tail instability is decreased, while the relaxation time of the beam is increased. Moreover, due to their interactions with the density inhomogeneities, Langmuir waves with larger $k$ can transfer part of their energy to Langmuir waves with smaller $k$, which in turn can damp and accelerate beam electrons of velocities $v>v_{b}$ up to $2 v_{b}$ and more. Note also that in plasmas with $\Delta n \gtrsim 3\left(v_{T} / v_{b}\right)^{2}$ Langmuir waveforms tend to form spatially localized and clumpy wave packets.

The presence of fluctuating density gradients generating various processes of wave reflection, refraction, and scattering as well as wave energy focusing alter the development of parametric instabilities occurring in plasmas with longwavelength density inhomogeneities; some of the first reasons are, for example, the random variations of the wave frequencies and thus of the resonance conditions between waves, $\omega_{k}=\omega_{k^{\prime}}+\omega_{k^{\prime \prime}}$, and the modification of the distribution of Langmuir wave energy in the $k$-space. Thus, the influence of $\Delta n \gtrsim 3\left(v_{T} / v_{b}\right)^{2}$ on the nonlinear dynamics of waves during Langmuir turbulence is important, as revealed by the following simulation results.

Figure 6 shows the time evolution of the Langmuir wave spectral energy $W_{L}$ and of the beam electron velocity distribution $f(v)$ in a plasma with $\Delta n \simeq 0.01$, near the threshold $3\left(v_{T} / v_{b}\right)^{2}$. One observes the presence of accelerated electrons (right panel) and the linked saturation of the wave energy growth (left panel, to compare with Figure 1).

Profiles of the Langmuir wave energy $|E|^{2}$ and the density fluctuations $\delta n / n_{0}$ along the simulation box of length $L$ are presented in Figure 7 for three different times $\omega_{p} t=30000$, 36000 , and 52000. One can see that Langmuir wave packets are focused and localized within a wide space region where the density fluctuations are forming a well (upper panels). Note that such observations were also done for density perturbations forming humps and not only depletions, as in the case presented here. After some time (middle panels) the local structure of the Langmuir waves, which form a set of more or less separated packets, is conserved despite the modification of the density profile. However, after the appearance of IS waves of noticeable intensity, the structure of the Langmuir packets becomes much more irregular and chaotic in the space region where short-scale density oscillations are rising (lower panels). This is due to the decay of Langmuir waves and to the consequent redistribution of energy between them, as shown below.

The spectra of the Langmuir and IS waves, shown in Figure 8 for the same times as in Figure 7, present features similar to those observed in Figure 4 for the case of small $\Delta n \simeq 0.001$; indeed, one can observe peaks corresponding to the development of a decay instability. However, due to the presence of randomly varying density inhomogeneities, some differences exist between the theory of decay in quasihomogeneous plasmas and the observations, as will be discussed below.

First, the wavenumber of the Langmuir mode observed near $k \lambda_{\mathrm{D}} \simeq-0.05$ at $\omega_{p} t \simeq 30000$ and 36000 is consistent with the value expected for a first decay cascade, i.e., 

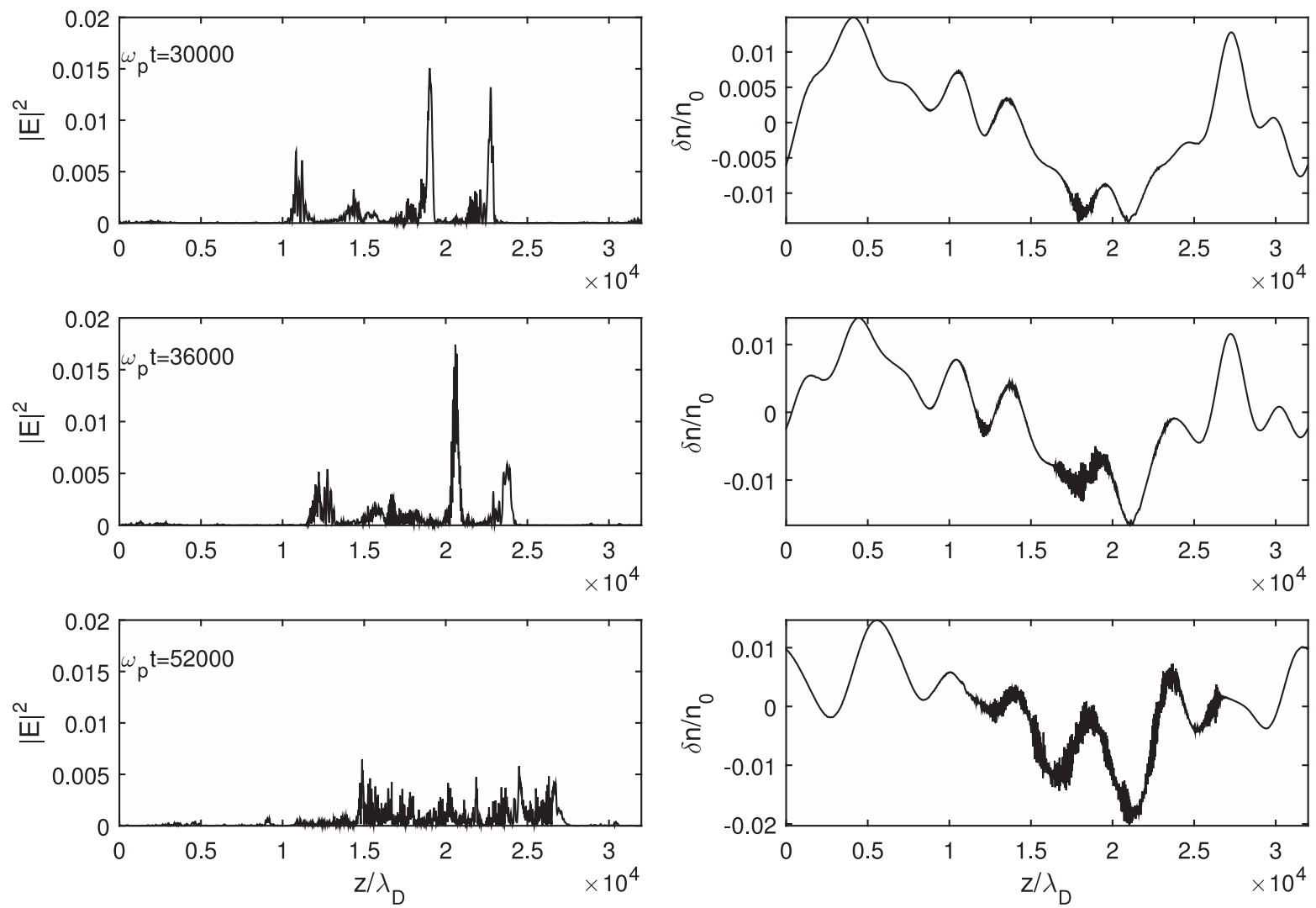

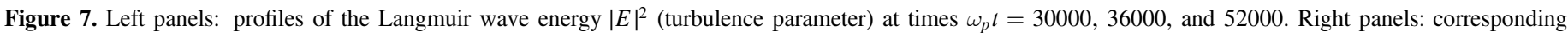

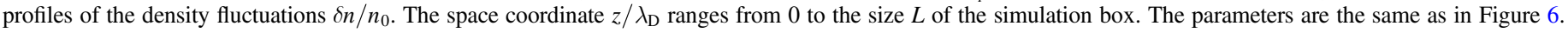
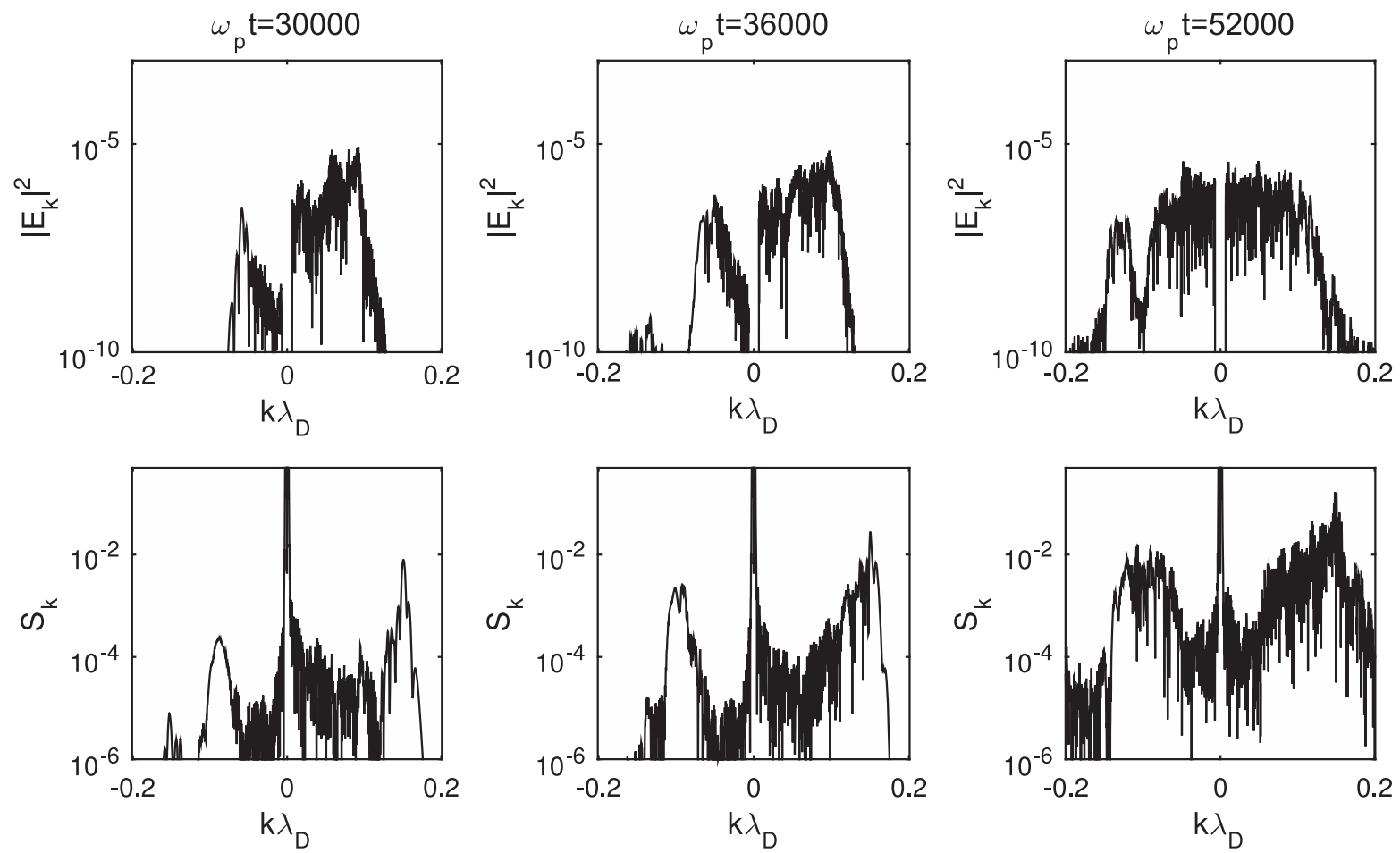

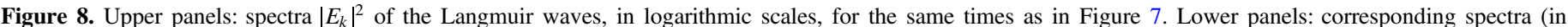

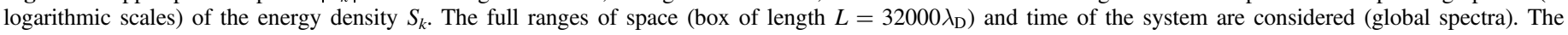
parameters are the same as in Figure 6. 

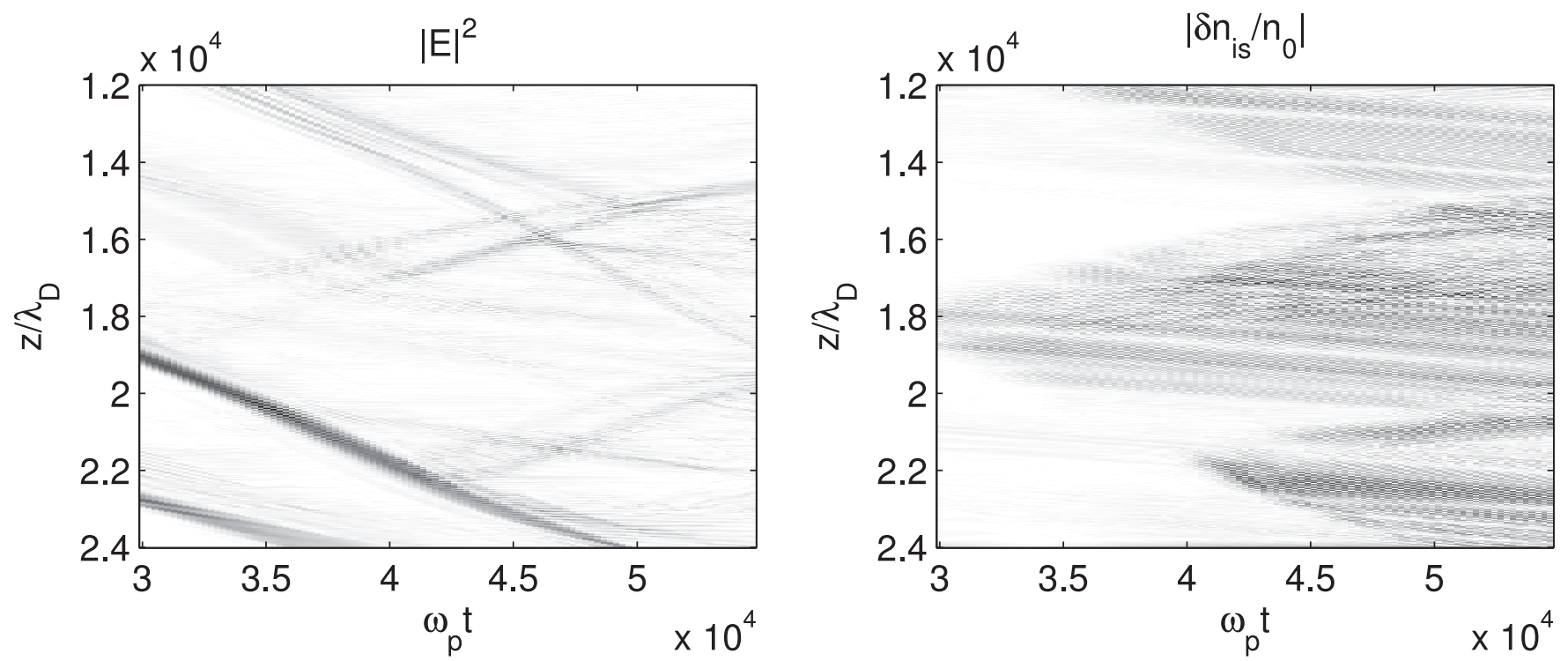

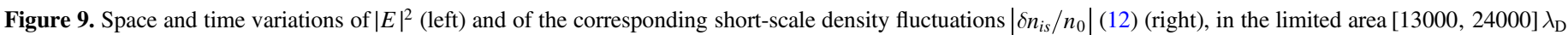
and during the time interval $30000<\omega_{p} t<54000$. The parameters are the same as in Figure 6 .

$k_{L^{\prime}} \lambda_{\mathrm{D}} \simeq\left(k_{0}-k_{L}\right) \lambda_{\mathrm{D}} \simeq-0.06 ;$ the value of $k_{L}$ is determined by the location of the highest peak with $k>0$ in the Langmuir spectrum at $\omega_{p} t \simeq 30000$ (upper left panel, Figure 8), i.e., $k_{L} \lambda_{\mathrm{D}} \simeq 0.09$ (here $k_{0} \lambda_{\mathrm{D}} \simeq 0.03$ and $k_{b} \lambda_{\mathrm{D}} \simeq 0.055$; note that the most excited Langmuir waves have phase velocities $\omega_{k} / k \leqslant v_{b}$, so that $\left.k_{L}>k_{b}\right)$. At the same time, the largest peak in the IS spectrum is centered near $k \lambda_{\mathrm{D}} \simeq 0.15$, which is very close to the expected wavenumber $k_{S^{\prime}} \lambda_{\mathrm{D}} \simeq$ $\left(2 k_{L}-k_{0}\right) \lambda_{\mathrm{D}} \simeq 0.15$ of IS waves produced during a first decay cascade. Moreover, the peak at $k \lambda_{\mathrm{D}}=0.025$ in the Langmuir spectrum (upper left and middle panels) represents the mode $k_{L^{\prime \prime}} \lambda_{\mathrm{D}}=\left(k_{L^{\prime}}-k_{S^{\prime \prime}}\right) \lambda_{\mathrm{D}}=\left(k_{L}-2 k_{0}\right) \lambda_{\mathrm{D}} \simeq 0.03$ generated during a second decay cascade, for which the corresponding IS wavenumber is expected at $k_{S^{\prime \prime}} \lambda_{\mathrm{D}}=$ $\left(-2 k_{L}+3 k_{0}\right) \lambda_{\mathrm{D}} \simeq-0.09$, which is close to the mode at $k \lambda_{\mathrm{D}} \simeq-0.1$ visible in the IS spectrum (lower middle panel). Let us stress that the complex peak structure in the lowfrequency spectra can be attributed to the fact that several decay instability regions exist and interfere one with another. Moreover, effects due to Langmuir waves' reflections on the density humps are essential and lead to the widening of the peaks in the corresponding wave spectra.

Second, one important difference compared to the quasihomogeneous plasma case is that wave decay occurs in localized space-time regions. Indeed, for a given moment in the time range when decay occurs, there are not many occurrences of decay spread accross the full range of $z$ (as in Figure 3), but the development of such a process can be observed only in two or three localized space regions. In this case, the processes of scattering, reflection, and/or 1D refraction modify the waves' propagation locally, depending, for example, on the presence along the waves' paths of more or less sharp gradients, deep wells, or wide humps, so that these waves can lose some part of their energy during their propagation, reducing by this fact the possibility of being submitted to further decay processes along $z$. To understand how a locally arising nonlinear interaction of Langmuir waves with density fluctuations develops, let us focus our attention on finite portions $\left[z_{1}, z_{2}\right]$ of the simulation box. Figure 9 shows the spatio- temporal evolution of the Langmuir wave energy $|E|^{2}$ and the short-scale IS density fluctuations $\delta n_{i s} / n_{0}$ in the subbox $\left[z_{1}, z_{2}\right]=[13000,24000] \lambda_{\mathrm{D}}$, during the time interval $30000 \lesssim \omega_{p} t \lesssim 54000$. One can see the formation of three main regions of IS wave activity (right panel), which broaden and eventually intersect. Note the emergence of IS waves traveling in the direction opposite to the beam propagation with a group velocity around $-c_{s} / v_{T}$, starting, for example, at $z \simeq 21000 \lambda_{\mathrm{D}}$ and $\omega_{p} t \simeq 45000$ : they correspond to the development of a second cascade of decay instability (see also above).

The left panel shows that the interactions of Langmuir packets with density fluctuations occur during limited and rather short time durations. Then, as their group velocity $v_{g} / v_{T}$ significantly exceeds the IS velocity $c_{S} / v_{T}$, the Langmuir waves can propagate away from these interaction regions; further, part of their energy can be transferred to Langmuir packets arising from a first decay cascade and propagating in the opposite direction, with a group velocity $-v_{g} / v_{T}$ (see, for example, the waves crossing near $z \simeq 21000 \lambda_{\mathrm{D}}$ and $\omega_{p} t \simeq 47000$ in the left panel). The beatings between Langmuir waves propagating in opposite directions lead to the generation of IS waves. Processes involving such beatings form a part of the cascade of energy transfer in the weak turbulence theory; however, some differences exist compared to the homogeneous plasma case: due to the presence of background density fluctuations, the decay processes are occurring locally, i.e., only in specific time and space regions.

Figure 10 shows the "local" wave spectra corresponding to Figure 9, i.e., computed within a specific subbox [13000, $24000] \lambda_{\mathrm{D}}$ during a limited time $30000 \lesssim \omega_{p} t \lesssim 54000$. Those reveal more clearly than the global ones-corresponding to the full space-time domain (Figure 8) - the development of a cascade of energy transfer during the interaction of Langmuir and IS waves. This is particularly visible in the low-frequency spectra, with peaks appearing clearly in the vicinity of $k \lambda_{\mathrm{D}}=0.15$ and -0.1 . In the high-frequency spectra, peaks are excited near $k \lambda_{\mathrm{D}}=-0.065,0.025,0.065$ and 0.09 (upper left panel); the largest one, which corresponds to beam-driven 

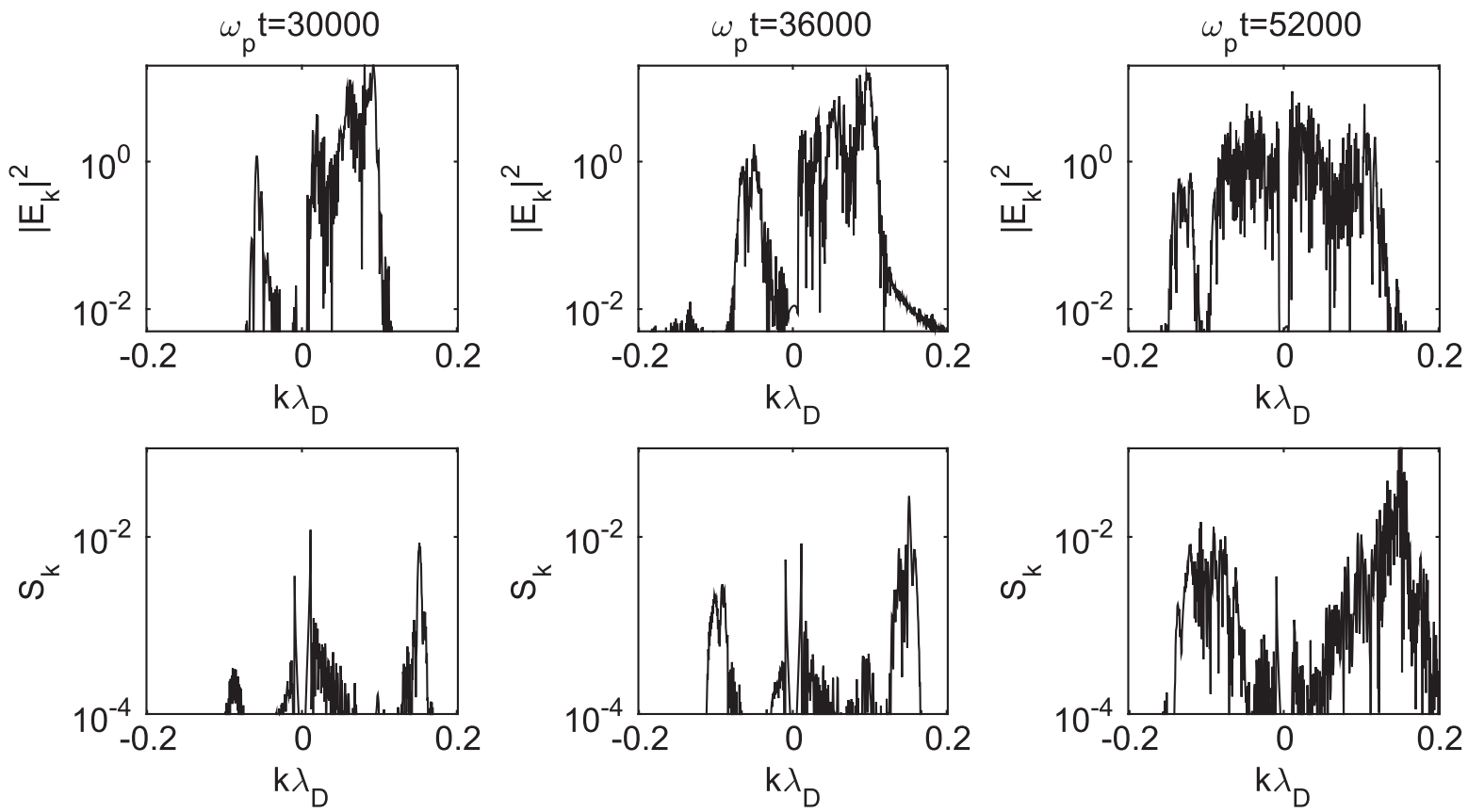

Figure 10. Upper panels: "local" spectra $\left|E_{k}\right|^{2}$ of the Langmuir waves, in logarithmic scales, for the same times as in Figures 7 and 8 . Lower panels: corresponding "local" spectra (in logarithmic scales) of the energy density $S_{k}$. All spectra are computed in the limited space-time area corresponding to [13000, 24000] $\lambda_{\mathrm{D}}$ and $30000<\omega_{p} t<54000$. The parameters are the same as in Figure 6.
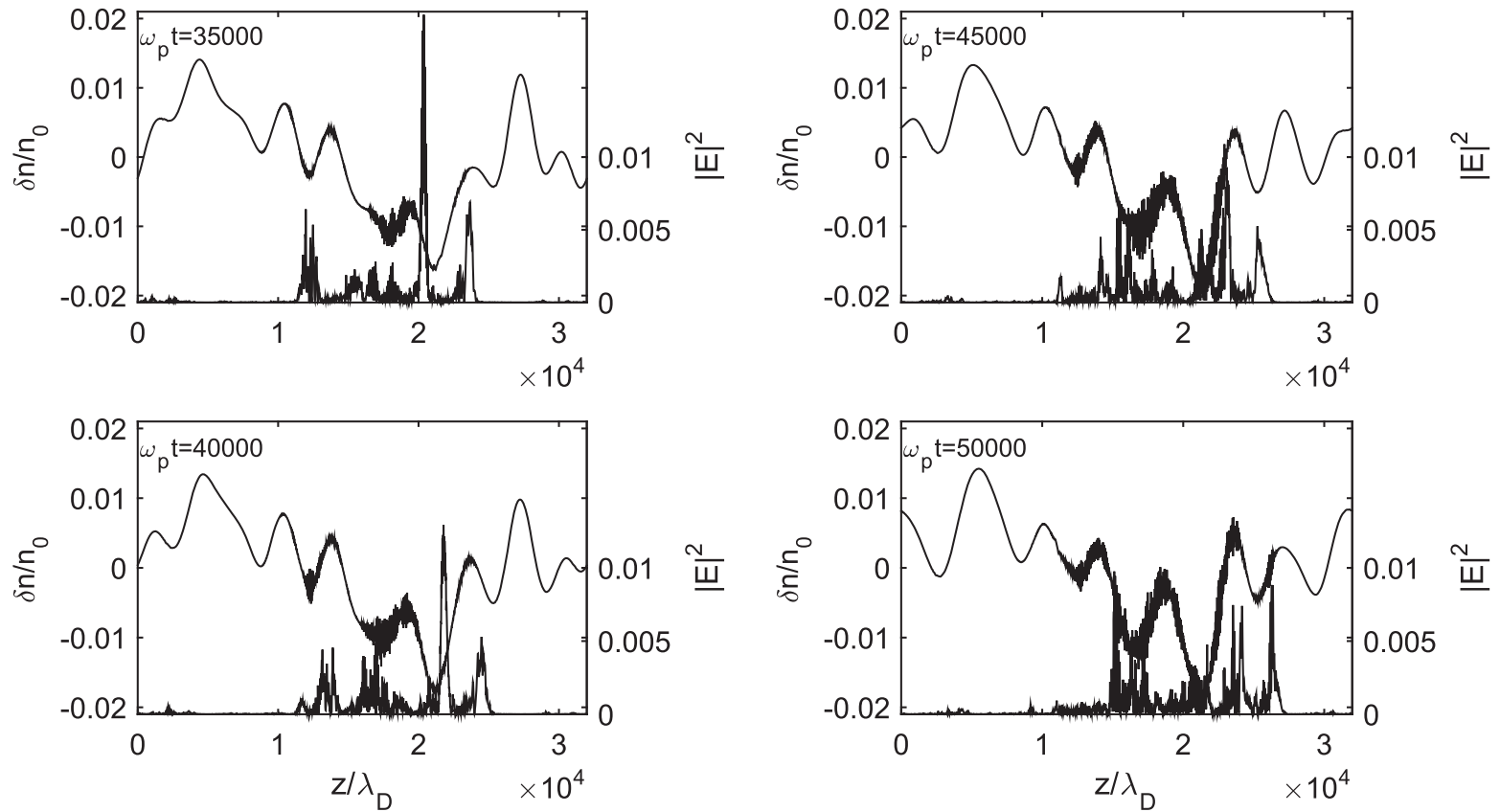

Figure 11. Profiles of the normalized Langmuir wave energy density $|E|^{2}$ (right axis and lower curves in each panel) superposed to the density fluctuations $\delta n / n_{0}$ (left axis and upper curves), in the area [13000 $\left.\lambda_{\mathrm{D}}, 24000 \lambda_{\mathrm{D}}\right]$, at times $\omega_{p} t=35000,40000,45000$, and 50000. Note that the origin of both vertical axes are not coinciding. The parameters are the same as in Figure 6.

waves, is located at $k \lambda_{\mathrm{D}} \simeq 0.09$. As discussed above, a first decay cascade of the Langmuir wave $k_{L} \lambda_{\mathrm{D}} \simeq 0.09$ starts, giving rise to a counterpropagating Langmuir wave at $k_{L^{\prime}} \lambda_{\mathrm{D}} \simeq\left(k_{0}-k_{L}\right) \lambda_{\mathrm{D}} \simeq-0.06$ and to an IS wave with $k_{S^{\prime}} \lambda_{\mathrm{D}}=\left(2 k_{L}-k_{0}\right) \lambda_{\mathrm{D}} \simeq 0.15$. Further (at $\left.\omega_{p} t \gtrsim 30000\right)$, a second decay cascade occurs, providing the peak at $k_{L^{\prime \prime}} \lambda_{\mathrm{D}}=\left(k_{L}-2 k_{0}\right) \lambda_{\mathrm{D}} \simeq 0.03$ (upper right panel) and an IS wave at $k_{S^{\prime \prime}} \lambda_{\mathrm{D}}=\left(-2 k_{L}+3 k_{0}\right) \lambda_{\mathrm{D}} \simeq-0.09$, propagating opposite to the beam. We obviously recover the same results as above, but with significantly less ambiguity in the interpretation of the spectral peaks.

Figure 11 shows the profiles of the Langmuir wave energy $|E|^{2}$ and the density fluctuations $\delta n / n_{0}$ within the same spatiotemporal area as in Figures 9-10. IS waves' short-scale oscillations appear first at $\omega_{p} t=35000$ within the region $17000 \lambda_{\mathrm{D}}<z<20000 \lambda_{\mathrm{D}}$; further they extend over wider space domains, eventually occupying for $\omega_{p} t \gtrsim 50000$ the subarea $[13000,24000] \lambda_{\mathrm{D}}$. However, they do not extend over 


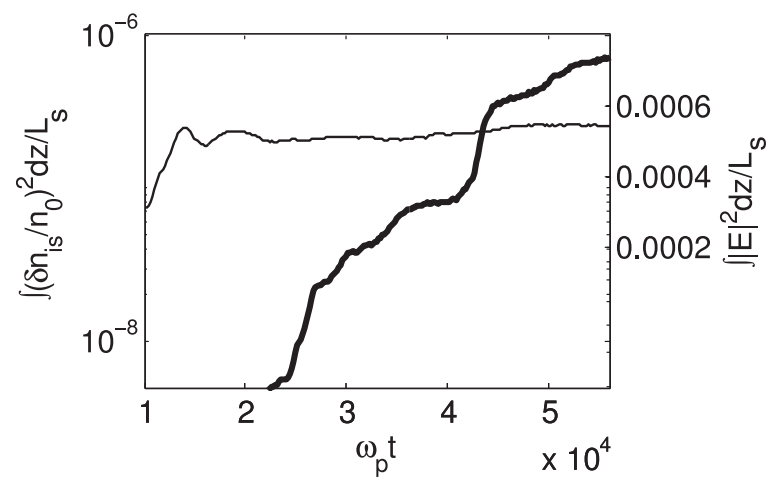

Figure 12. Time variations of the energy densities of the IS short-scale oscillations (bold line, left axis) and of the Langmuir waves (thin line, right axis), i.e., $\int_{L_{s}}\left|\delta n_{i s} / n_{0}\right|^{2} d z / L$ and $\int_{L_{s}}|E|^{2} d z / L$, respectively, in logarithmic scales; the energies are computed within a localized area $L_{s}$ of the simulation box, in the range $15000 \leqslant \omega_{p} t \leqslant 55000$. The main parameters are the following: $n_{b} / n_{0}=510^{-5}, v_{b} / v_{T}=14, \Delta n \simeq 0.02, L=10000 \lambda_{\mathrm{D}}$.

the whole space profile (see also Figure 7). One can observe that the various space regions of IS waves' generation are separated one from the other. Moreover, in each area where these waves appear, the growth of their energy stops when the Langmuir packets have traveled away and left the interaction region due to the large difference between the IS and the Langmuir waves' group velocities, $v_{g} \gg c_{s}$ (compare, for example, the panels at $\omega_{p} t=35000$ and $\left.\omega_{p} t=40000\right)$. The escaping Langmuir waves can then interact with IS waves in other space-time regions where such oscillations appear.

Figure 12 shows the time variation of the IS and Langmuir waves' energies, $\int_{L_{s}}\left|\delta n_{i s} / n_{0}\right|^{2} d z / L_{s}$ and $\int_{L_{s}}|E|^{2} d z / L_{s}$, respectively, obtained by simulations involving a plasma with density fluctuations of larger average level, i.e., $\Delta n \simeq 0.02$ (other parameters being the same as in Figure 1) and computed in the limited domain $L_{s}=\left[13000 \lambda_{\mathrm{D}}, 24000 \lambda_{\mathrm{D}}\right]$. One can see that, while the plasma waves' energy varies only slightly, the energy of the IS short-scale oscillations $\delta n_{i s} / n_{0}$ exhibits two periods of exponential growth; each of them corresponds to the growth of a localized IS wave packet rising in a specific region of the subbox $L_{s}$. The first period, between $\omega_{p}(t) \simeq 24000$ and $\omega_{p} t \simeq 28000$, corresponds to the crossing of two Langmuir packets of positive but different group velocities; indeed, when a Langmuir packet passes through a density hump, its group velocity can be significantly reduced due to $1 \mathrm{D}$ refraction effects. The second period, i.e., $42000 \lesssim \omega_{p} t \lesssim 46000$ (see also Figure 9), corresponds to the occurrence of a first cascade of Langmuir waves' decay, whose growth rate can be estimated as $\quad \Gamma=2.7 \quad 10^{-4} \omega_{p} \quad$ (with $\quad \gamma_{D} \simeq 4 \quad 10^{-4} \omega_{p}$; ; see Equations (13)-(14)).

Let us present in this case a typical example of Langmuir decay involving several cascades. Figure 13 shows the spatiotemporal variation of the Langmuir wave energy density $|E|^{2}$ and of the short-scale density oscillations $\delta n_{i s} / n_{0}$, in the subbox $[5000,8000] \lambda_{\mathrm{D}}$ and the time interval $4500 \leqslant \omega_{p} t \leqslant 9000$, when the beam instability is saturated. In the left panel, beamdriven Langmuir waves are propagating with $v_{g}>0$ through the region [5000, 6700] $\lambda_{\mathrm{D}}$ for $\omega_{p} t \lesssim 58000$; near $\omega_{p} t \simeq 58300$ and $z \simeq 6800 \lambda_{\mathrm{D}}$, their amplitudes are strongly enhanced when they cross another Langmuir packet traveling with $v_{g}<0$. At the same time, IS waves propagating with the group velocity $c_{S} / v_{T}>0$ are excited (right panel).
In Figure 14, which shows the corresponding profiles of $|E|^{2}$ and $\delta n / n_{0}$ (including $\delta n_{i s} / n_{0}$ ) at six moments of time, one can see that $|E|^{2}$ reaches its maximum when the waves approach the local density hump near $z \simeq 6800 \lambda_{\mathrm{D}}$ (left middle panel at $\omega_{p} t \simeq 58300$ ); at this point, both packets propagating in opposite directions interact and, as a result, some large part of the Langmuir energy is reflected and propagates away with $v_{g}<0$ (see Figure 13, left, and Figure 14, top right). A similar event can be observed later, near $\omega_{p} t \simeq 60000$ and $z \simeq 7300 \lambda_{\mathrm{D}}$ (Figure 13, left): the Langmuir wave decays. Figure 14 at $\omega_{p}(t) \simeq 60600$ and 63000 shows how the counterpropagating packet separates itself from the packet with $v_{g}>0$, eventually taking with it almost all the Langmuir energy. The two peaks at $\omega_{p} t \simeq 63000$ (Figure 14 , bottom right) can be clearly identified in Figure 13 as the two packets propagating with $v_{g}<0$, in the time range $60000 \lesssim \omega_{p} t \lesssim 65000$. Moreover, near $\omega_{p} t \simeq 66000$ and $z \simeq 6000 \lambda_{\mathrm{D}}$, a second decay cascade occurs, giving rise to Langmuir packets propagating with $v_{g}>0$ and IS waves' oscillations propagating with $-c_{S} / v_{T}<0$ (Figure 13).

It is well known from theory (Musher et al. 1995) that decay processes involve several cascades of energy transfer to waves with longer wavelengths. The successive occurrence of two decay cascades was discussed and presented above for the cases of very small or finite average levels of external density fluctuations, i.e., for $\Delta n \simeq 0.001$ and $\Delta n \simeq 0.01$ (see, e.g., Figure 10). However, one must stress that decay cascading in a plasma with background density fluctuations of finite $\Delta n$ presents specific features, which we examine now in more detail on the basis of simulation results presented in Figures 12-14. Note first that according to the decay resonance conditions, only three cascades are expected to occur for the beam and plasma parameters considered here. During these successive processes, Langmuir wavenumbers decrease more and more, starting from the value $k_{L} \lambda_{\mathrm{D}} \simeq 0.11$ corresponding to the most excited beam-driven Langmuir waves (not shown here); then, as a consequence of the resonance conditions, the Langmuir wave products of the first, second, and third decay cascades are characterized by the following wavenumbers: $k_{L^{\prime}} \lambda_{\mathrm{D}} \simeq$ $\left(k_{0}-k_{L}\right) \lambda_{\mathrm{D}} \simeq-0.08, \quad k_{L^{\prime \prime}} \lambda_{\mathrm{D}} \simeq\left(k_{L}-2 k_{0}\right) \lambda_{\mathrm{D}} \simeq 0.05$, and $k_{L^{\prime \prime}} \lambda_{\mathrm{D}} \simeq\left(3 k_{0}-k_{L}\right) \lambda_{\mathrm{D}} \simeq-0.02$, whereas for the IS daughter waves we have $k_{S^{\prime}} \lambda_{\mathrm{D}} \simeq\left(2 k_{L}-k_{0}\right) \lambda_{\mathrm{D}} \simeq 0.19, \quad k_{S^{\prime \prime}} \lambda_{\mathrm{D}} \simeq$ $\left(-2 k_{L}+3 k_{0}\right) \lambda_{\mathrm{D}} \simeq-0.13$, and $k_{S^{\prime \prime \prime}} \lambda_{\mathrm{D}} \simeq\left(2 k_{L}-5 k_{0}\right) \lambda_{\mathrm{D}} \simeq$ 0.07 , respectively. A fourth cascade is not possible as the resonance conditions are not fulfilled. An essential point lies in the fact that the Langmuir waves $\mathcal{L}^{\prime \prime}$ coming from the second cascade propagate in the direction of the beam drift and have larger phase velocities than those excited first by the beam instability. So the second decay cascade can play an important role in the acceleration of beam electrons above the initial beam velocity $v_{b}$.

The first two decay cascades are very clearly observed in Figures 13-14. For $\omega_{p} t \gtrsim 56000$ and $z \lesssim 6800 \lambda_{\mathrm{D}}$, two wave packets propagate along the beam direction $\left(v_{g}>0\right)$ and a third one in the opposite direction $\left(v_{g}<0\right)$ (Figure 13, left). In the vicinity of $z \simeq 6900 \lambda_{\mathrm{D}}$, the packet with $v_{g}>0$ and with the largest amplitude collides near $\omega_{p} t \simeq 58000$ with the packet propagating with $v_{g}<0$ (see also Figure 14 at $\omega_{p} t \simeq 58300$ ). IS waves are generated as a result of the beating between these two colliding packets (Figure 13, right); during this process, the weaker amplitude packet with $v_{g}<0$ gets energy from the more intense one with $v_{g}>0$. This mechanism could be 

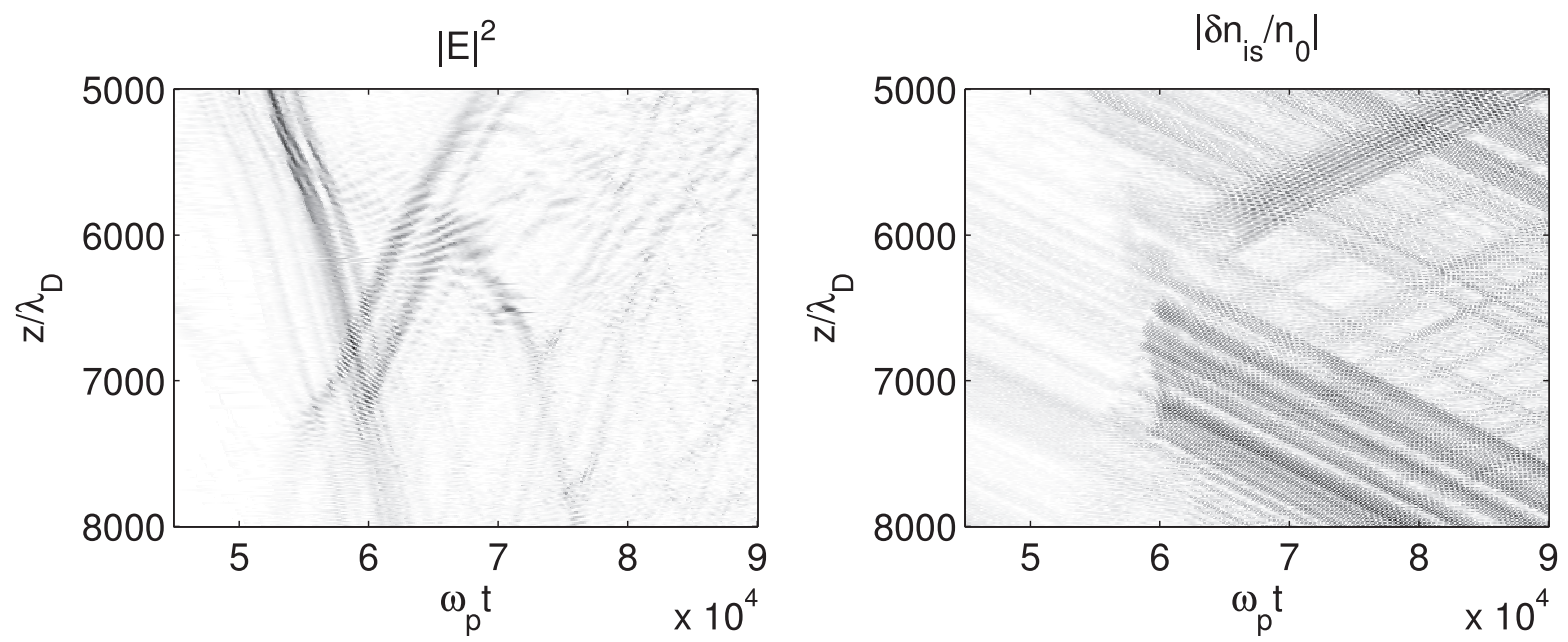

Figure 13. Space and time variations of $|E|^{2}$ (left) and of the corresponding short-scale density fluctuations $\delta n_{i s} / n_{0}$ (right), in the area [5000, 8000] $\lambda_{\mathrm{D}}$. The parameters are the same as in Figure 12.
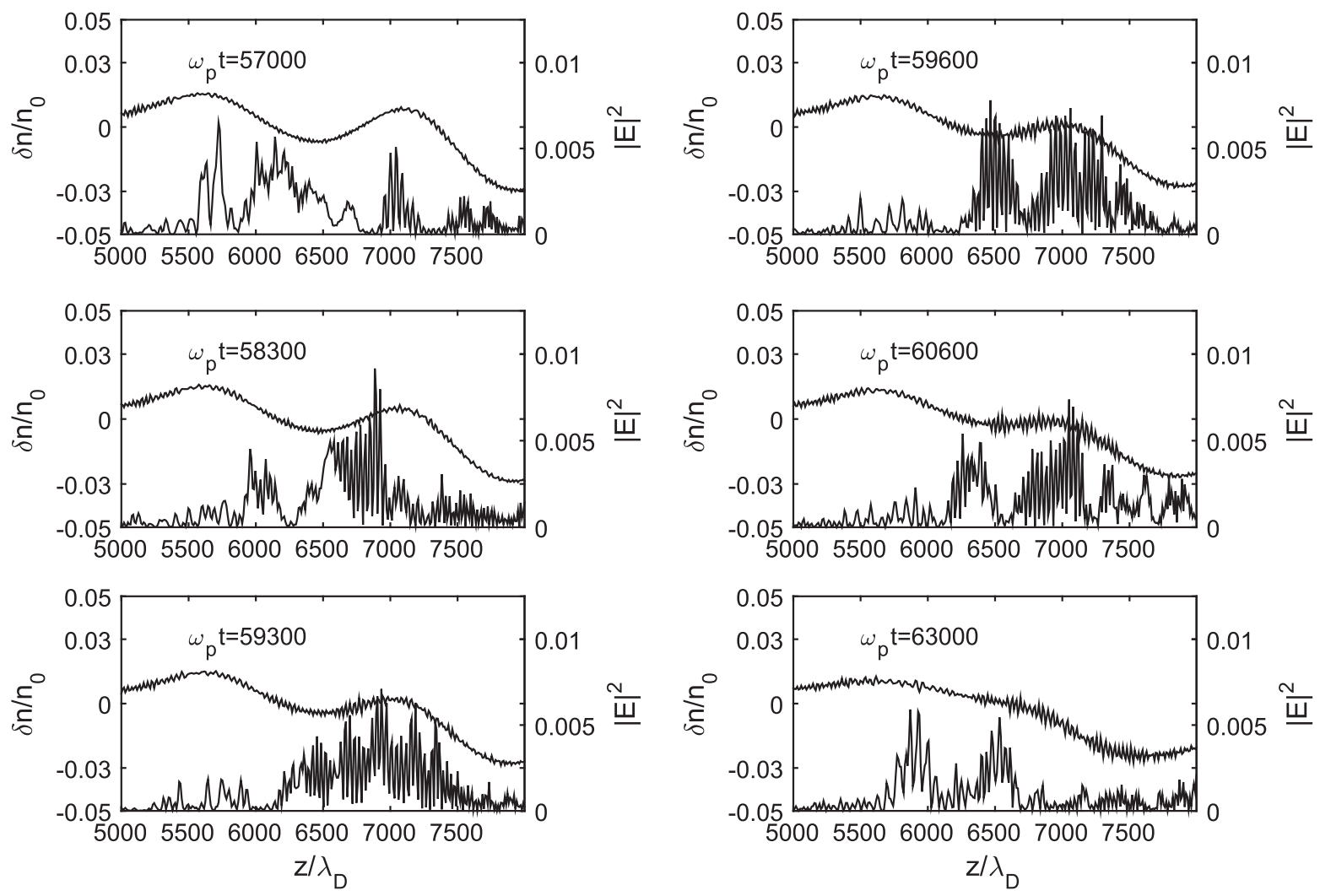

Figure 14. Profiles of the Langmuir wave energy density $|E|^{2}$ (right axis and lower curves in each panel) superposed to the density fluctuations $\delta n / n_{0}$ (left axis and upper curves), in the area $\left[13000 \lambda_{\mathrm{D}}, 24000 \lambda_{\mathrm{D}}\right]$, at times $\omega_{p} t=57000,58300,59300,59600,60600$, and 63000 . Note that the zero of both vertical axes are not coinciding. The parameters are the same as in Figure 12.

considered as the first cascade of wave energy transformation, but the actual situation is more complicated. Indeed, almost simultaneously, the colliding packet with $v_{g}>0$ reflects on the density hump presenting a maximum at $z \simeq 7000 \lambda_{\mathrm{D}}$ (Figure 14 at $\omega_{p} t \simeq 58300$ ). The propagating second packet with $v_{g}>0$ consequently collides with the amplified packet with $v_{g}<0$ and with the reflected one (Figure 13, left; Figure 14 at $\omega_{p} t \simeq 59300$ ). Their beatings also generate IS waves. At $\omega_{p} t \gtrsim 60000$ two wave packets are traveling in the direction opposite to the beam propagation (Figure 14 at $\omega_{p} t \simeq 63000$ ). Later, near $\omega_{p} t \simeq 67000$ and $z \simeq 6000 \lambda_{\mathrm{D}}$, a process starts which can be called the second decay cascade, involving one of these packets and generating IS waves propagating in the negative direction (at group velocity $-c_{s} / v_{T}$ ) with no signatures of any Langmuir wave reflection (Figure 13, left). At last, near $\left(\omega_{p} t \simeq 73500, z \simeq 6800 \lambda_{\mathrm{D}}\right)$ and $\left(\omega_{p} t \simeq 76000\right.$, $z \simeq 7800 \lambda_{\mathrm{D}}$ ), structures similar to a third decay can be observed on the space-time evolution pattern (Figure 13, left); one can indeed observe a small but noticeable enhancement of the IS wave emission along the direction of the beam propagation, near $\omega_{p} t \simeq 73500$ and $z \simeq 6800 \lambda_{\mathrm{D}}$ (Figure 13, right), which is expected from a third cascade; however, as the 

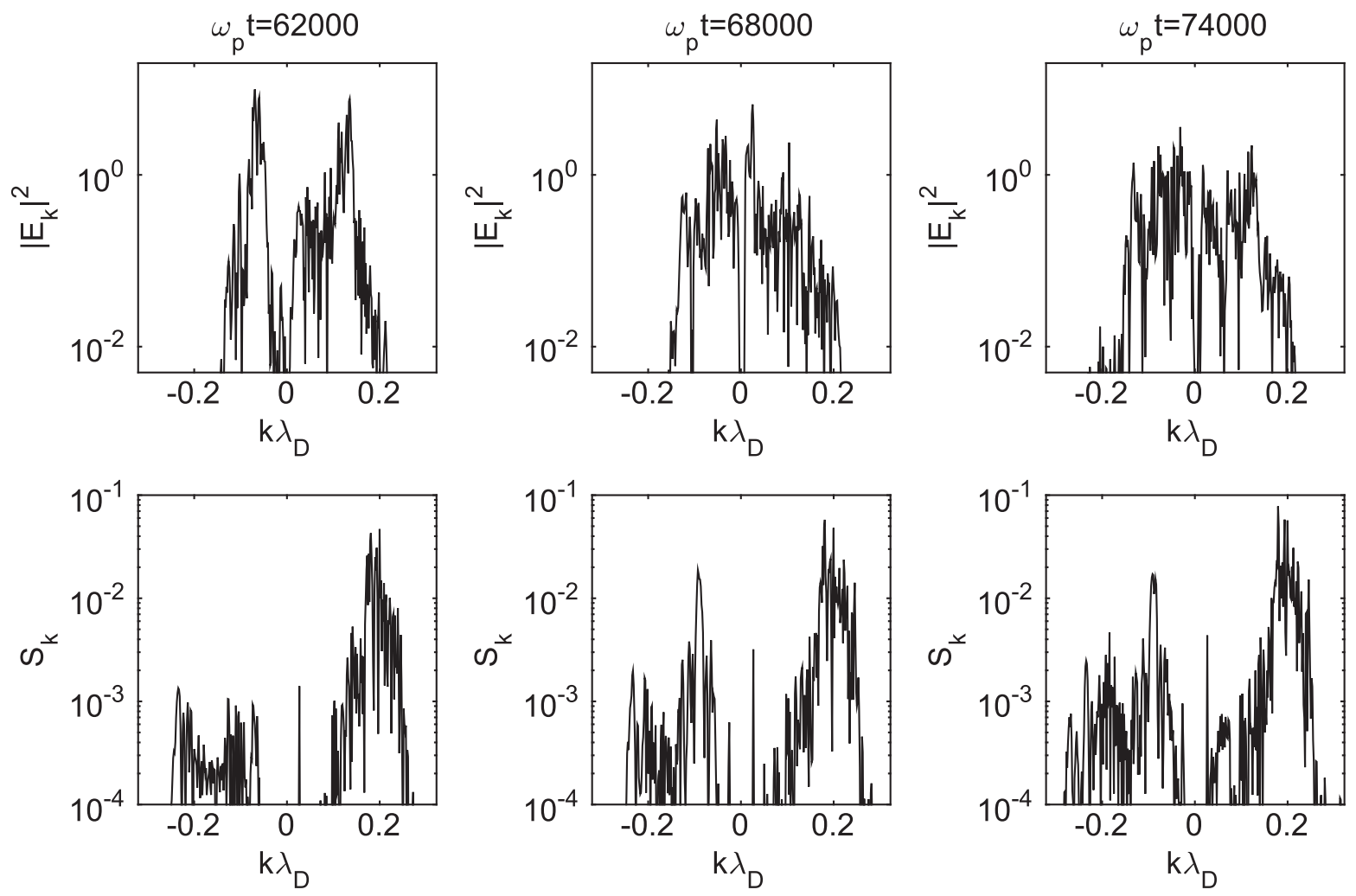

Figure 15. Local spectra (computed in the area $[4500,8500] \lambda_{\mathrm{D}}$ ) of Langmuir and ion sound waves for three moments of time $\omega_{p} t=62000,68000$, and 74000 . The parameters are the same as in Figure 14.

wave packets have amplitudes that are too weak, we are not able to state that this process is actually and certainly a third cascade. Indeed, Figure 15 presents the corresponding local spectra of Langmuir and IS waves at the three time moments $\omega_{p} t=62000,68000$, and 74000; they show that if peaks at $k_{L^{\prime \prime}} \lambda_{\mathrm{D}} \simeq-0.02$ (upper right panel) and $k_{S^{\prime \prime}} \lambda_{\mathrm{D}} \simeq 0.07$ (lower right panel) are visible, they are either too weak (IS spectrum) or mixed with other effects (Langmuir spectrum). However, at $\omega_{p} t=62000$ and $\omega_{p} t=68000$, the position of some peaks in the IS and Langmuir spectra are very close to those predicted by the resonant wave decay theory in homogeneous plasmas for the first and the second cascades (see the discussion in the previous paragraph). As mentioned above, the spectrum of Langmuir waves for $\omega_{p} t=74000$ does not show clearly distinguishable peaks, which can be explained by two effects, i.e., the propagation of the waves in the inhomogeneous plasma and their exchanges of energy with the beam. At the same time, three main peaks can be observed in the IS spectrum: two correspond to the wavenumbers of the IS wave products of the two first decay cascades, i.e., $k_{S^{\prime}} \lambda_{\mathrm{D}} \simeq 0.19$ and $k_{S^{\prime \prime}} \lambda_{\mathrm{D}} \simeq-0.13$, whereas the third one near $k \lambda_{\mathrm{D}} \simeq-0.2$ is likely due to a nonresonant wave-wave interaction.

Finally, it is important to stress at this stage that it is generally very difficult, if not impossible, to separate the processes of wave reflection on density fluctuations from that of parametric wave-wave interactions; both effects are usually working together in a inhomogeneous plasma, and the origin of the counterpropagating (i.e., with $v_{g}<0$ ) Langmuir waves produced cannot be determined conclusively in most cases. Note also that reflections of Langmuir waves on longwavelength density inhomogeneities can, in some conditions, favor the emergence of nonlinear decay processes, owing to the appearance of parametric interaction processes involving waves traveling in the direction opposite to the beam propagation. One must also take into account the significant role of the variations of the long-wavelength density fluctuations due to their own dynamics; for example, the local positive gradient of the density hump in Figure 14 disappears for $\omega_{p} t \gtrsim 63000$ and conditions for IS wave generation in the vicinity of this region become less favorable than when Langmuir energy can focus on the gradients of the humps near some reflection points.

\section{DISCUSSION AND CONCLUSION}

Simulations have shown that three-wave decay processes including several cascades can occur in inhomogeneous plasmas as those of the solar wind in the source regions of type III bursts and, in particular, in the course of Langmuir turbulence in the presence of electron beams. Wave-wave interaction processes where a Langmuir wave decays into a Langmuir and where IS waves can be observed in plasmas with average levels of density fluctuations up to a few percent. Decay has been notably identified by the simultaneous presence of peaks in the high- and low-frequency wave energy spectra, at the wavenumbers of the mother and the daughter waves, in agreement with the waves' resonance conditions. Moreover, the growth rate of the IS energy has been shown to fit with the predictions of the parametric decay theory for homogeneous plasmas and monochromatic waves, at least up to $\Delta n \simeq 0.02$. A very good agreement between the wavenumbers predicted by this theory and our simulations of Langmuir turbulence in inhomogeneous plasmas has been observed. This is due to the fact that the waves' dispersion equations are not significantly modified by the irregularities and that the 
wavenumbers' shifts due to the density fluctuations are smaller than the spectral widths of the peaks.

For small values of $\Delta n$ below the threshold, i.e., $\Delta n \ll 3\left(v_{T} / v_{b}\right)^{2}$, three-wave decay processes occur commonly during Langmuir turbulence and can arise after some time in all space regions; moreover, they can be described with a rather good agreement by the weak turbulence theory for monochromatic waves in homogeneous plasmas. In this case, Langmuir turbulence does not present focused peaks along the space profiles (with a lack of Langmuir energy in some regions), but the wave energy is distributed roughly uniformly along the simulation box: wave decay is not a localized process and can evolve in time until its own saturation. On the contrary, when $\Delta n \gtrsim 3\left(v_{T} / v_{b}\right)^{2}$, several other effects such as wave scattering, reflection, or $1 \mathrm{D}$ refraction, for example, can compete with the decay processes whose duration is more limited and which take place locally in space-time locations where the physical conditions allow their appearance. These specific regions can be of various nature and are not only concerning the vicinity of reflection points on the density fluctuations' gradients where the Langmuir energy can be accumulated and focused before the decays start. In this case, as the Langmuir group velocity $v_{g}$ is generally much larger than the IS group velocity $c_{s}$, the decay processes are not stopped due to nonlinear wave saturation but to kinematic effects, when the fastest Langmuir packets escape the localized regions where they locally interact with IS waves. However, in the asymptotic stage of the system's evolution, short-scale IS oscillations can exist along the whole space profile, due to the fact that $v_{g}$ is significantly larger than $c_{s}$ and that the Langmuir packets have time to travel through the density profile several times during the entire time of the simulation. Moreover, IS oscillations survive for a long time and can interact several times with the same Langmuir packets passing again in their neighborhood or with other arriving ones.

Generally, the larger $\Delta n$ is (and/or the smaller the wavelengths of the fluctuations), the more decay processes become rare, as they can be overcome by scattering processes of waves on the density inhomogeneities. For large values of $\Delta n$ lying significantly above the threshold (i.e., $0.03 \lesssim \Delta n \lesssim 0.06$ ), decay cannot be observed or only rarely and under specific local conditions; in this case, the scattering of the waves on the inhomogeneities, which occurs on a much shorter timescale than the decay process, tends to destroy the coherency between the waves necessary for decay. For example, the occurrence of wave decay is observed in our simulations for $\Delta n \simeq 0.03$, even if this process is mostly overcome by reflection phenomena; however, for $\Delta n>0.03$, no example of wave decay could be found for the physical parameters used.

While decay processes in quasi-homogeneous plasmas are more likely to occur when the beam is almost fully relaxed, such effects can start in a inhomogeneous plasma well before the beam is relaxed due to reflection processes and to the accumulation of energy near the reflection points on the density profiles. Indeed, the interferences between Langmuir waves can become more frequent because the possibilities of generating counterpropagating Langmuir waves are enhanced due to the presence of density fluctuations. Then, in some cases, reflection processes can favor the appearance of a decay process, i.e., increase its efficiency in its early stage, due to the coupling of Langmuir waves with amplitudes above the thermal level.

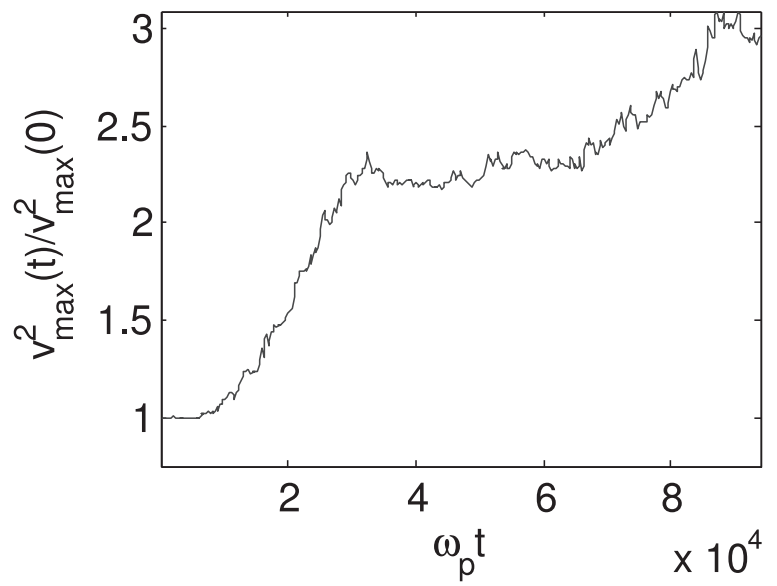

Figure 16. Variation with time of the maximum (normalized) square velocity of the population of accelerated particles; a second increase occurs near $\omega_{p} t \simeq 67000$, when the second decay cascade starts. The parameters are the same as in Figure 12.

Langmuir wave decay allows the transfer of energy to smaller $k$ waves, which can in turn accelerate beam electrons and generate high-energy tails. In particular, if the first decay cascade provides waves propagating in the direction opposite to the beam, the second cascade's Langmuir products propagate along the beam direction and thus can interact with beam particles of velocities larger than the initial beam velocity $v_{b}$. However, they usually have small $k$ and phase velocities around or exceeding $2 v_{b}$, so that all of them cannot be in resonance with the accelerated particles, whose largest velocity exceeds the velocity $2 v_{b}$ only for very long simulations. The next cascade processes can only shift the Langmuir waves to large resonance velocities and cannot affect the acceleration of beam particles. In the cases presented above, we can observe an increase with time of the largest velocity of the population of accelerated particles, beginning from $\omega_{p} t \simeq 67000$, when the second decay cascade starts (Figure 16; see also Figure 13). Such a process is, however, occurring in advanced stages of the beam relaxation, and thus is believed to play a minor role compared to the acceleration induced by the presence of randomly fluctuating density inhomogeneities. A more detailed study will be carried out in a forthcoming paper.

Finally, the occurrence of decay cascades has a noticeable influence on the redistribution of energy between the different $k$-scales of the Langmuir turbulence, leading to the appearance of specific modulation features in the corresponding Langmuir waveforms. Thus, wave-wave coupling can play a significant role in shaping these waveforms and producing the clumpy and characteristic modulation patterns observed by the satellites STEREO and WIND. Two examples are shown in Figure 17, for the parameters of Figures 12-16, i.e., when $\Delta n \simeq 0.02$. Let us simulate what would observe a virtual satellite starting at time $\omega_{p} t \simeq 50000$ and position $z_{S} \simeq 8000 \lambda_{\mathrm{D}}$ and moving with a velocity $v_{S}=0.1 v_{T}$; this satellite concludes its travel at $\omega_{p} t \simeq 68000$, arriving at the position $z \simeq 6200 \lambda_{\mathrm{D}}$; during its path, it passes through the region where the decay process occurs, i.e., within the space-time domain $58000 \lesssim$ $\omega_{p} t \lesssim 62000$ and $6300 \lambda_{\mathrm{D}} \lesssim z \lesssim 7300 \lambda_{\mathrm{D}}$ (see Figure 13). On the other hand, when the satellite starts at the same time but at another location $z_{S} \simeq 5700 \lambda_{\mathrm{D}}$, for example, and travels with the same velocity, it does not encounter any wave decay process, as such effect is not observed in the corresponding 

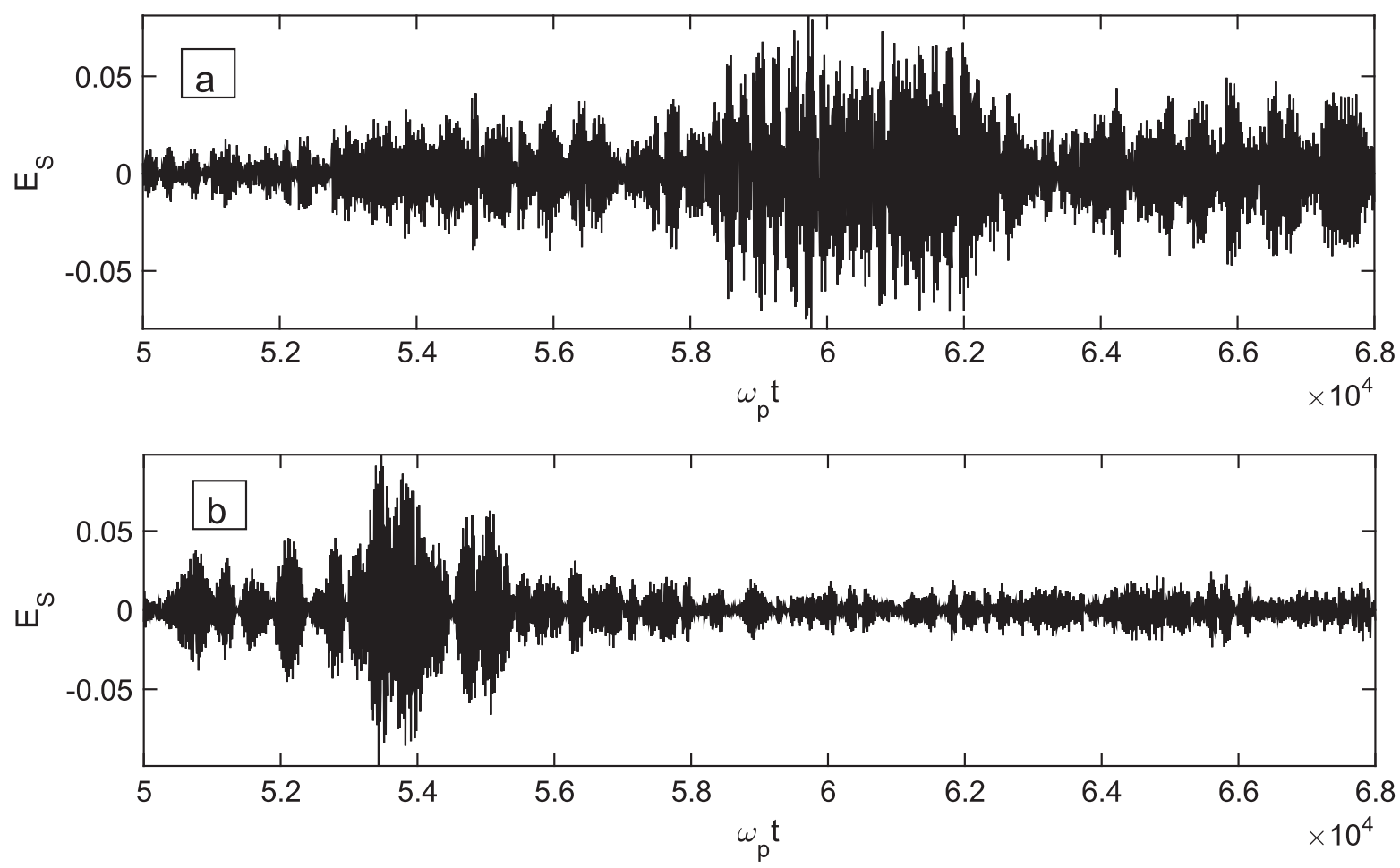

Figure 17. Langmuir waveforms (registered electric fields $E_{S}$ as a function of time) that would be observed by a satellite moving at a velocity $v_{S}=0.1 v_{T}$ and starting at positions $z_{S}=8000 \lambda_{\mathrm{D}}$ (upper panel) and $z_{S}=5700 \lambda_{\mathrm{D}}$ (lower panel) at time $\omega_{p} t=50000$; the satellite travels until the time $\omega_{p} t=68000$. The parameters are the same as in Figure 12.

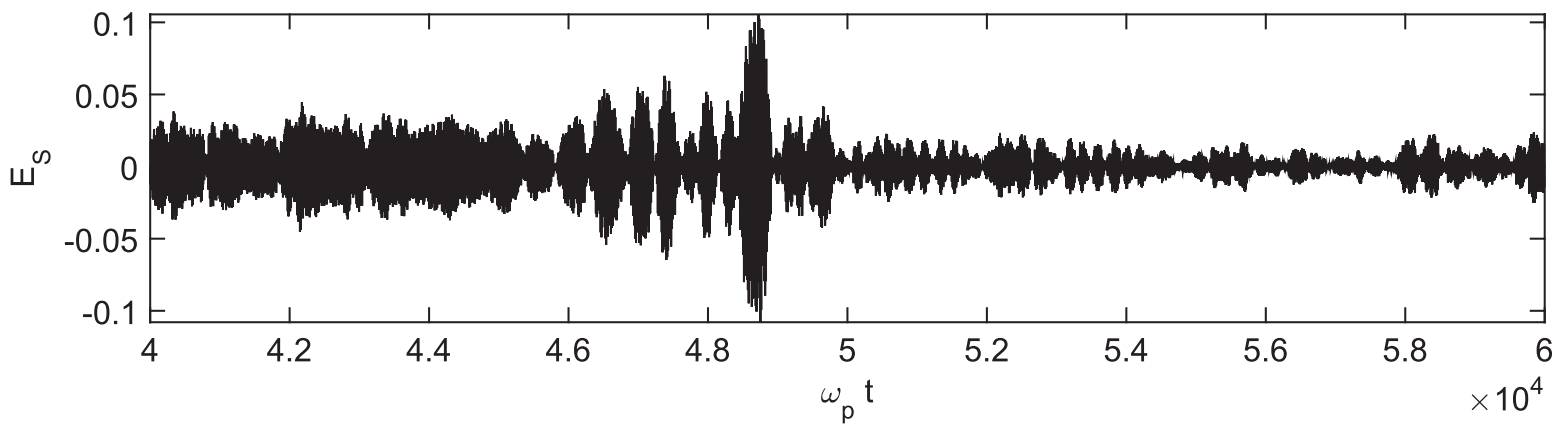

Figure 18. Langmuir waveform (registered electric field $E_{S}$ as a function of time in arbitrary units) that would be observed by a satellite moving at a velocity $v_{S}=0.15 v_{T}$ and starting at a position $z_{S}=7000 \lambda_{\mathrm{D}}$ at time $\omega_{p} t=40000$; the satellite travels until the time $\omega_{p} t=60000$. The parameters are the same as in Figure 12, but with $\Delta n \simeq 0.03$.

space-time range. Let us now compare in Figure 17 the waveforms observed by the virtual satellite for these two cases, respectively, i.e., the normalized electric fields $E_{S}(t)=\operatorname{Re} \sum_{k} E_{k}(t) \exp \left(i k\left(z-v_{S} t\right)-i \omega_{p} t\right)$ registered as a function of time (Krafft et al. 2014). One observes in the upper panel $\left(z_{S} \simeq 8000 \lambda_{\mathrm{D}}\right)$ that specific modulation features revealing wave-wave beating appear within the range $58000 \lesssim \omega_{p} t \lesssim 62000$, when the satellite travels from $z \simeq 7200 \lambda_{\mathrm{D}}$ to $z \simeq 6800 \lambda_{\mathrm{D}}$, which corresponds exactly to the space-time domain where decay occurs (see Figures 13-14).

On the contrary, this kind of modulated structures are not visible in the waveform when the satellite starts at $z_{S} \simeq 5700 \lambda_{\mathrm{D}}$ (lower panel), i.e., when it does not cross any decay event. Such a result is in good agreement with the observations by the satellite STEREO reported recently in Graham \& Cairns (2013), where their Figure 4 shows Langmuir waveforms observed before and after an electrostatic decay, in conditions very similar to ours (in particular, the electric field has a negligible perpendicular component); before decay, the amplitude of the waves varies smoothly and slowly, whereas after the decay a modulation pattern revealing wavewave beating can be observed.

Another example is shown in figure 18 for the case of a plasma with a larger average level of inhomogeneities, i.e., $\Delta n \simeq 0.03$, where the other parameters are similar to those of Figures 12-16. A satellite passes with the velocity $v_{S}=0.15 v_{T}$ through the region where the simulations evidenced a Langmuir decay. As a consequence, the waveform exhibits, during the time lapse $46000 \lesssim \omega_{p} t \lesssim 48500$ when the decay process occurs, modulation features characteristic of wave beating.

Finally, in order to fit more closely to realistic physical conditions, we have performed simulations including finite ion and electron damping factors calculated taking into account bulk plasmas with nonthermal electron and ion Kappa tails. 

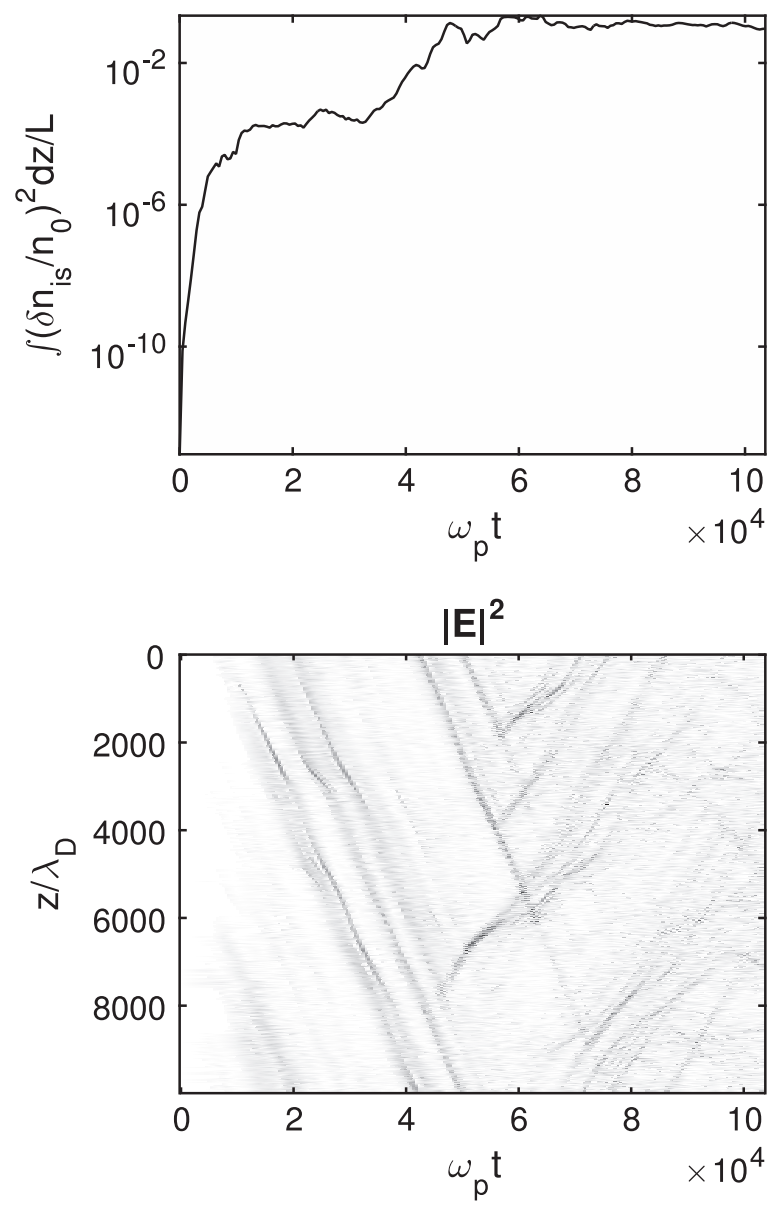

Figure 19. Upper panel: time variation of the energy densities of the IS shortscale oscillations. Lower panel: space and time variations of $|E|^{2}$, in the case when Landau damping on ions and electrons is taken into account, due to the presence of nonthermal Kappa tails in the bulk plasma velocity distribution. The main parameters are the following: $n_{b} / n_{0}=510^{-5}, v_{b} / v_{T}=14$, $\Delta n=0.01, L=10000 \lambda_{\mathrm{D}}$.

Figure 19 presents the time variation of the IS energy and the space-time evolution of the Langmuir energy density, respectively. The upper panel shows a significant growth of the IS energy near $\omega_{p} t \simeq 40000$ whereas, around this time, one can observe in the lower panel the appereance of backscattered Langmuir waves propagating with negative group velocities. Therefore, even for significant (but moderate, due to the typical electron to ion temperature ratios $T_{e} / T_{i} \gtrsim 3$ ) values of the ion and electron damping factors $\left|\gamma_{i}\right| \ll \omega_{S}$ and $\left|\gamma_{e}\right| \ll \omega_{p}$, damping effects due to the presence of nonthermal electron and ion tails do not suppress the occurence and the fulfillment of the Langmuir wave decay processes. Moreover, those can even be identified more clearly due to the more or less efficient suppression of some wavelengths in the spectra.

This work was granted access to the HPC resources of IDRIS under the allocation 2013- i2013057017 made by GENCI. This work has been done within the LABEX Plas@ par project, and received financial state aid managed by the Agence Nationale de la Recherche, as part of the programme "Investissements d'avenir" under the reference ANR-11IDEX-0004-02. C.K. acknowledges the "Programme National Soleil Terre" (PNST) and the Centre National d'Etudes
Spatiales (CNES, France). V.K. acknowledges the financial support from CNES through the grant "STEREO invited scientist."

\section{REFERENCES}

Bale, S. D., Burgess, D., Kellogg, P., et al. 1996, GeoRL, 23, 109

Bale, S. D., Larson, D. E., Lin, R. P., et al. 2000, JGR, 105, 27353

Bougeret, J.-L., Goetz, K., GoldsteinKaiser, M. L., et al. 2008, SSRv, 136, 487

Breizman, B. N., \& Ruytov, D. D. 1970, JETP, 30, 759

Burinskaya, T. M., Rauch, J. L., \& Mogilevskii, M. M. 2004, PlPhR, 30, 756

Cairns, I. H. 2000, PhPl, 7, 4901

Cairns, I. H., \& Robinson, P. A. 1992, GeoRL, 19, 2187

Cairns, I. H., \& Robinson, P. A. 1995, ApJ, 453, 959

Celnikier, L. M., Harvey, C. C., Jegou, R., Moricet, P., \& Kemp, M. 1983, A\&A, 126, 293

Celnikier, L. M., Muschietti, L., \& Goldman, M. V. 1987, A\&A, 181, 138

Dudok de Wit, T., \& Krasnoselskikh, V. 1995, PhPl, 2, 4307

Ergun, R. E., Larson, D., Lin, R. P., et al. 1998, ApJ, 503, 435

Ergun, R. E., Malaspina, D. M., Cairns, Iver H., et al. 2008, PhRvL, 101, 051101

Escande, G. V., \& de Genouillac, D. F. 1978, A\&A, 68, 405

Galeev, A. A., \& Sagdeev, R. Z. 1975, in Reviews of Plasma Physics, Vol. 7, ed. M. A. Leontovich (New York: Consultants Bureau)

Galeev, A. A., Sagdeev, R. Z., Shapiro, V. D., \& Shevchenko, V. I. 1977, JETP, 45, 266

Gibson, S. E., Newman, D. L., \& Goldman, M. V. 1995, PhRvE, 52, 558

Graham, D. B., \& Cairns, I. H. 2013, JGR, 118, 3968

Gurnett, D. A., Hospodarsky, G. B., Kurth, W. S., Williams, D. J., \& Bolton, S. J. 1993, JGR, 98, 5631

Gurnett, D. A., Kurth, W. S., Shaw, R. R., et al. 1992, SSRv, 60, 341

Gurnett, D. A., Maggs, J. E., Gallagher, D. L., Kurth, W. S., \& Scarf, F. L. 1981, JGR, 86, 8833

Henri, P., Briand, C., Mangeney, A., et al. 2009, JGR, 114, A03103

Henri, P., Califano, F., Briand, C., \& Mangeney, A. 2010, JGR, 115, A06106

Hess, S. L. G., Malaspina, D. M., \& Ergun, R. E. 2011, JGR, 116, A07104

Hospodarsky, G. B., \& Gurnett, D. A. 1995, GeoRL, 22, 1161

Hospodarsky, G. B., Gurnett, D. A., Kivelson, M. G., et al. 1994, JGR, 99 , 13363

Huang, Y., \& Huang, G.-L. 2008, ChA\&A, 32, 178

Kadomtsev, B. B. 1965, Plasma Turbulence (London, New York: Academic)

Kellogg, P. J. 1986, A\&A, 169, 329

Kellogg, P. J., Goetz, K., Lin, N., et al. 1992, GeoRL, 19, 1299

Kellogg, P. J., Goetz, K., Monson, S. J., et al. 2009, JGR, 114, A02107

Kellogg, P. J., Goetz, K., Monson, S. J., \& Bale, S. D. 1999, JGR, 104, 17069

Kontar, E. P., \& Pecseli, H. L. 2002, PhRvE, 65, 066408

Krafft, C., \& Volokitin, A. 2006, PhPl, 13, 122301

Krafft, C., \& Volokitin, A. 2010, PhPl, 17, 102303

Krafft, C., \& Volokitin, A. 2013, PhLA, 377, 1189

Krafft, C., \& Volokitin, A. 2014, EPJD, 68, 370

Krafft, C., Volokitin, A., \& Zaslavsky, A. 2005, PhP1, 12, 112309

Krafft, C., Volokitin, A., \& Zaslavsky, A. 2010, PhRvE, 82, 066402

Krafft, C., Volokitin, A. S., \& Krasnoselskikh, V. V. 2013, ApJ, 778, 111

Krafft, C., Volokitin, A. S., Krasnoselskikh, V. V., \& Dudok de Wit, T. 2014, JGR, 119, 9369

Li, B., Willes, A. J., Robinson, P. A., \& Cairns, I. H. 2003, PhP1, 10, 2748

Lin, R. P., Levedahl, W. K., Lotko, W., Gurnett, D. A., \& Scarf, F. L. 1986, ApJ, 308, 954

Lin, R. P., Potter, D. W., Gurnett, D. A., \& Scarf, F. L. 1981, ApJ, 251, 364

Malaspina, D. M., Cairns, I. H., \& Ergun, R. E. 2010, JGR, 115, A01101

Musher, S. L., Rubenchik, A. M., \& Zakharov, V. E. 1995, PhR, 252, 177

Nicholson, Dwight R., \& Goldman, Martin V. 1978, PhFl, 21, 1766

Nulsen, A. L., Cairns, I. H., \& Robinson, P. A. 2007, JGR, 112, A05107

O’Neil, T. M., Winfrey, J. H., \& Malmberg, J. H. 1971, PhFl, 14, 1204

Robinson, P. A., \& Newman, D. L. 1991, JGR, 96, 17733

Robinson, P. A., Willes, A. J., \& Cairns, I. H. 1993, ApJ, 408, 720

Rubenchik, A. M., \& Shapiro, E. G. 1993, JETP, 76, 48

Ryutov, D. D. 1970, JETP, 30, 131

Sagdeev, R. Z., \& Galeev, A. A. 1969, Nonlinear Plasma Theory (New York: Benjamin)

Souček, J., Krasnoselskikh, V., Dudok de Wit, T., Pickett, J., \& Kletzing, C. 2005, JGR, 110, A08102 
Thejappa, G., MacDowall, R. J., \& Bergamo, M. 2013, AnGeo, 31, 1417

Thejappa, G., MacDowall, R. J., Scime, E. E., \& Littleton, J. E. 2003, JGR, 108, 1139

Umeda, T., \& Ito, T. 2008, PhPl, 15, 084503

Vedenov, A. A., Gordeev, A. V., \& Rudakov, L. I. 1967, PIPh, 9, 719

Vedenov, A. A., \& Ryutov, D. D. 1975, Reviews of Plasma Physics, Vol. 6 (New York: Consultants Bureau)

Vedenov, A. A., Velikhov, E. P., \& Sagdeev, R. Z. 1961, NucFu, 1, 82

Volokitin, A., \& Krafft, C. 2004, PhP1, 11, 3165
Volokitin, A., \& Krafft, C. 2012, PPCF, 54, 085002

Volokitin, A., Krasnoselskikh, V., Krafft, C., \& Kuznetsov, E. 2013, in AIP Conf. Proc., 1539, Modelling of the Beam-Plasma Interaction in a Strongly Inhomogeneous Plasma (Melville, NY: AIP), 78

Zakharov, V. E. 1972, JETP, 35, 908

Zakharov, V. E., Musher, S. L., \& Rubenchik, A. M. 1985, PhR, 129, 285

Zaslavsky, A., Krafft, C., \& Volokitin, A. 2006, PhRvE, 73, 016406

Zaslavsky, A., Krafft, C., \& Volokitin, A. 2007, PhP1, 14, 122302

Ziebell, L. F., Gaelzer, R., \& Yoon, P. H. 2001, PhPl, 8, 3982 